\title{
Heterogeneous Beliefs and Imperfect Competition in Sequential Auction Markets*
}

\author{
Hervé Boco ${ }^{\dagger}$, Laurent Germain ${ }^{\ddagger}$, Fabrice Rousseau ${ }^{\S}$
}

$1^{\text {st }}$ November 2014

${ }^{*}$ We are grateful to the seminar participants at Toulouse Business School, at the Smurfit Business School, Paris Dauphine University, MFA Meetings in Orlando and Luxembourg School of Finance, and at the ESSEC for their comments. We are particularly grateful to Shmuel Baruch, Gilles Chemla, Bernard Dumas, Laurence Lescourret, Sebastien Pouget, Jean Charles Rochet and Mehmet Saglam for their useful comments. Finally, we would like to thank Hadria Hatim for his excellent research assistance.

${ }^{\dagger}$ Hervé Boco Toulouse University, Toulouse Business School, 20 Boulevard Lascrosses 31000 Toulouse France, h.boco@esc-toulouse.fr.

${ }^{\ddagger}$ Laurent Germain Toulouse University, Toulouse Business School, 20 Boulevard Lascrosses 31000 Toulouse France, l.germain@esc-toulouse.fr.

${ }^{\S}$ Fabrice Rousseau Maynooth University Department of Economics, Finance and Accounting Maynooth Co. Kildare Ireland, Fabrice.Rousseau@nuim.ie. 


\begin{abstract}
This paper analyzes a multi-auction setting in which informed strategic agents are endowed with heterogeneous noisy signals about the liquidation value of a risky asset. One result is that when the variance of the noise is small the competition between traders takes the form of a rat race during all the periods of trading. As we increase the level of the noise in the traders' signals, a waiting game phase appears and the intensity of the rat race, observed only at the last auctions, decreases. In sharp contrast with the previous literature, when the variance of the noise is very large, we only observe a waiting game.
\end{abstract}

JEL Classification: G14-G24-D43-D82

Keywords: efficiency, asymmetric information, noise, liquidity, adverse selection, competition. 


\section{Introduction}

Analyzing the strategic trading behavior of informed traders in a dynamic setting is the focus of a large body of literature in Finance. Different frameworks are used in order to perform that task. The seminal paper by Kyle (1985) examines the trading behavior of a single perfectly informed trader. The paper shows that the monopolistic trader will limit the size of his early trades in order not to reveal too much information too early. This leads to information being gradually incorporated into prices. That result depends on some crucial assumptions such as the presence of a single informed trader but also on the structure of the private information i.e. whether it is perfect or not. Holden and Subrahmanyam (1992) show, to the contrary of Kyle (1985), that the competition resulting from the presence of more than one informed trader with identical information results in almost all the private information to be revealed in the early auctions. ${ }^{1}$ Foster and Viswanathan (1996) analyze the case of imperfect competition when the traders' information is correlated. Aggregate private information gives then the liquidation value of the risky asset. Back, Cao and Willard (2000) study the competition between strategic traders in continuous time. Both papers show that the result of the competition between informed traders is very complex and depends critically on the initial correlation between the informed traders' signals.

In our paper, we revisit the competition between heterogeneously informed strategic traders. However, as in Admati and Pfleiderer (1988), we assume that each trader is endowed with a noisy normally distributed signal about the liquidation value of the risky asset $\tilde{v}$. This allows us to know the structure of information of all trades.

Given that framework, we derive the unique linear equilibrium in a multi-auction market where traders receive heterogenous signals. It allows us to study the convergence of prices to the liquidation value of the risky asset, knowing the exact structure of the heterogeneity in the beliefs of the insiders.

Our article aims at answering the following questions:

\footnotetext{
${ }^{1}$ Michener and Tighe (1991), Foster and Viswanathan (1993) find the same result.
} 
- What dynamic strategies should informed market participants use to maximize their profits? How does the reaction of the informed agents evolve over time according to their private signals and prices?

- How quickly does the price adjust to reflect the presence of informed traders?

- How are the insiders' profits affected by noisy private signals? Can informed traders reduce competition when they have noisy private signals i.e. can noisy information be profitable for informed traders?

- Is there an optimal level of noise that maximize traders' profits?

Considering the effect of the variance of the noise on the traders' behavior is not equivalent to considering the effect of the correlation between signals. A change in the correlation between signals only measures the degree to which signals are identical or not. A change in the variance of the noise in the traders' signals does not only affect the correlation between the traders' signals, but also the correlation of the traders' signals with the liquidation value of the asset. Then, changing the level of the noise simultaneously affects the correlation between the traders' signals and the correlation with the liquidation value of the asset. Indeed, a large variance of the noise leads to a lower correlation between signals. This implies a reduction of the level of competition by giving each trader a monopolistic position on his private information and thus prevents competition from destroying his profits. However, it also implies that informed agents are trading on noise which in turn reduces their expected profits. This effect is not captured by looking at the effect of the correlation between signals.

Furthermore, our framework enables us to study the trade-off between noise and competition in a dynamic setting highlighted in Dridi and Germain (2009). ${ }^{2}$ When the number of traders is greater than four and for some values of the noise, they obtain that the traders get more profits with noisy information than with perfect information leading to noise

\footnotetext{
${ }^{2}$ The model of Dridi and Germain (2009) is a particular case of our model corresponding to a static setting.
} 
acting as a commitment not to trade. In our paper, we also study that trade-off in a dynamic setting and, as such competition encompasses two dimensions: one temporal i.e. the number of auctions and one spatial i.e. the number of informed traders.

Our main results are the following:

- The existence of a linear equilibrium is not always guaranteed. We find that the trading process continues as long as informed traders retain some private information not incorporated in the market maker's information set. We also find that the values of the noise (measured by the variance of noise in the traders signal) that guarantee the existence of an equilibrium are limited by the level of competition between traders.

- When the competition is strong (the number of informed traders and/or the number of auctions is high), increasing the noise in the traders' private information leads to higher profits than when that noise is small. In that case, traders can make more profits with noisy information than with perfect information. However, adding too much noise in the traders' signals always decreases their profits. When the level of competition is not as strong, noise always reduces the profit of the informed traders. These results generalize the findings of Dridi and Germain (2009) previously highlighted.

- The optimal noise (i.e. maximizing the informed traders' expected profits) increases with the number of traders and with the number of auctions. Moreover, as in Dridi and Germain (2009) the optimal aggregate profit has a strictly positive finite limit when the number of traders is large.

- The optimal individual profit has a strictly positive finite limit when the number of auctions is large.

The highlighted trade-off between noise and competition bears some similarities with the results put forward by Foster and Viswanathan (1996) and Back et al. (2000) regarding the level of correlation of the signals and the expected profits of the traders. Indeed, Foster 
and Viswanathan (1996) show that the expected profits of the traders are higher when there is some positive correlation compared to the case where the signals are uncorrelated. In our model, we concentrate on the level of noise and noise diminishes the correlation between private signals. We also show that noise can, in some cases, increase expected profits.

Ostrovsky (2012) highlights that in dynamics models the most important issue is the aggregation of information. In Foster and Viswanathan (1996) and Back, Cao and Willard (2000) the dispersed information forms a sufficient statistic and prices converge to the liquidation value. In our model, prices do not, in general, converge to the liquidation value of the risky asset. However, we show that, when the number of informed traders increases indefinitely, prices tend to converge to the liquidation value. Moreover, the rate of the increase for the price informativeness is lower in our model than in Holden and Subrahmanyam (1992), as noise slows down the revelation of information.

Both Foster and Viswanathan (1996) and Back et al. (2000) find that market competition depends on the initial correlation between the informed traders' signals. When the correlation is not too strong, the competition has two phases. Firstly, insiders trade very aggressively and release much of their private information in the earlier trading periods. This phase is known as the "rat race". Secondly, since the correlation between the residual private information of the informed traders evolves over time, after a number of auctions the insiders' residual information is negatively correlated between each other. This reflects a difference of opinion between the informed agents about the final value of the risky asset. The informed participants then become more reluctant to trade, since each insider could be on the wrong side of the market. Hence, the trading activity is less intense. This phase is known as the "waiting game". During that phase, insiders conceal their private information. This phenomenon leads to an adverse selection problem in the market at the end of the trading day. Hence, the competition between the insiders does not automatically lead to more efficient prices as one approaches the time of liquidation. We also show that it is possible to have the reverse sequence of the two stages (first a rat race and then a waiting game). 
Looking at the trader's behavior, we obtain the following results. When the trader's private information is quite precise (strong correlation between the trader's signal), the dynamic competition between traders takes the form of a rat race (where traders trade very aggressively on their private information) during all the periods of trading. This result generalizes the findings of Holden and Subrahmanyam (1992), for the range of low levels of noise. ${ }^{3}$ However, when the private information is very noisy we only obtain a waiting game. This result is in sharp contrast to Foster and Viswanathan (1996). Indeed, they find that the waiting game is followed by a rat race. In this case, the insiders limit their orders since their private information is noisy. The waiting game observed in our model is not due to a negative correlation between the signals as a consequence of trading. We show that, in our model, the waiting game phase appears when the correlation between the signals of the traders is low - but positive.

Some papers have empirically investigated the competition between informed traders taking place in financial markets. Ellison and Mullin (2007) find that the information is gradually incorporated into price confirming the result found in Kyle (1985). Cho (2007) analyses the behavior of stock prices ahead of earnings announcements. The paper finds evidence of informed trading. However, the evidence is more consistent with Foster and Viswanathan (1996) than with Kyle (1985). Our model predicts that changes in volume during the trading day can be explained by the presence of noise in the information of the traders when they compete in the market.

The remainder of the paper is organized as follows. Section 2, presents the general setup. We show in section 3 the existence and the uniqueness of a linear equilibrium and characterize the different parameters at each auction. In section 4 , we study the informativeness, the market depth and the expected profits according to the level of noise in the signals of the informed traders, the number of auctions and the number of traders. Finally, in section 5, we make some concluding remarks. All proofs are gathered in the

\footnotetext{
${ }^{3}$ We show that the models of Kyle (1985) (discrete setting) and Holden and Subrahmanyam (1992) are encompassed in our model leading to the same results for some particular parameters values.
} 
Appendix.

\section{The Model}

We follow, in this paper, the notation of Kyle (1985) and Holden and Subrahmanyam (1992). We assume that a risky security is traded during $N$ sequential auctions in a time interval which begins at $t=0$ and ends at $t=1$. Let $\Delta t$ be the time interval between the $n$th auction and the previous auction $\left(\Delta t=\frac{1}{N}\right)$. At $t=1$, the liquidation value of the asset is revealed. This liquidation value is denoted by $\tilde{v}$, with $\tilde{v} \sim N\left(\bar{v}, \sigma_{v}^{2}\right)$. For simplicity and without loss of generality, we assume $\bar{v}=0$. In each auction, the following market participants are present:

- $M$ risk-neutral informed traders. At $t=0$ each insider $i=1, \ldots, M$ receives a signal $\tilde{S}_{i}=\tilde{v}+\tilde{\epsilon}_{i}$ about the liquidation value of the risky asset, where $\tilde{\epsilon}_{i} \sim N\left(0, \sigma_{\epsilon}^{2}\right)$, for $i=1, \ldots, M$. Moreover, we assume that the error terms, $\tilde{\epsilon}_{i}$, are mutually independent. Informed participants receive heterogeneous signals as in Admati and Pfleiderer (1988).

- Several liquidity traders who submit orders at each auction. They do not possess any information about the fundamental value of the risky asset. We denote by $\Delta \tilde{u}_{n}$ their aggregate orders and we assume that $\left(\Delta \tilde{u}_{n}\right)$ are independently and identically normally distributed with zero mean and variance $\sigma_{u}^{2} \Delta t$. Also, we assume that $\Delta \tilde{u}_{n}$ and $\tilde{v}$ are independent.

- Competitive risk-neutral market makers who observe the aggregate orders, but who do not know whether these orders stem from liquidity traders or insiders, and set the price $p_{n}$, at each auction $n$ in a Bayesian way.

At the $n$th auction we denote $\Delta \tilde{X}_{n}$ as the aggregate order of all informed traders, and $\pi_{i, n}$ the total expected profit of informed trader $i$, for $i=1, \ldots, M$, from auction $n$ to auction $N$. 
Each risk neutral informed trader determines his optimal trading strategy by a process of backward induction in order to maximize his expected profits given his conjectures about the trading strategies of the other informed traders. We look for a linear equilibrium where each informed trader chooses an order which is linear in his private information and the previous public price.

Competition in market making drives the market makers' expected profits to zero, conditional on the aggregate submitted orders $\tilde{w}_{n}=\Delta \tilde{X}_{n}+\Delta \tilde{u}_{n}$. Thus, at the $n$th auction, $p_{n}$ equals the expected value of $\tilde{v}$ conditional on the information available. The market makers' prices are linear in the observed prevailing aggregate order flow and the previous public price.

\section{Equilibrium}

Let $\tilde{x}_{i n}$ be the order of agent $i$ at auction $n$. Given the linear assumption of the equilibrium, the order is such that $\hat{x}_{i n}=\left(\alpha_{i, n} \tilde{S}_{i}+\beta_{n} p_{n-1}\right) \Delta t$. After $n-1$ periods of trading, trader $i$ maximizes his remaining expected profit from the $n$th auction to the last one.

At the $n$th auction, the market maker receives the aggregate order flow $\tilde{w}_{n}=\sum_{i=1}^{M} \tilde{x}_{i n}+$ $\Delta \tilde{u}_{n}$. Based on this aggregate order flow, the market maker updates, in a Bayesian way, her estimate of the fundamental value of the risky asset:

$$
p_{n}=E\left[\tilde{v} \mid \tilde{w}_{1}, \ldots, \tilde{w}_{n-1}, \tilde{w}_{n}\right]=p_{n-1}+\lambda_{n} \tilde{w}_{n}
$$

where $\lambda_{n}$ is the liquidity parameter at the $n$th auction. It is the regression coefficient of $\tilde{v}$ on $\tilde{w}_{n}$ conditional on $\tilde{w}_{1}, \ldots, \tilde{w}_{n-1}$, the past trades. Hence, the price set by the risk neutral market makers, at each auction $n, p_{n}$, is equal to the conditional expectation of $\tilde{v}$ given the information generated by the aggregate order process up to time $t=\frac{n}{N}$.

Given the order form and the price function, the trader's conditional expected profit can be written as:

$$
E\left[\pi_{i n} \mid p_{0}, \ldots, p_{n-1}, \tilde{S}_{i}\right]=k_{1, n-1} \tilde{S}_{i}^{2}+k_{2, n-1} p_{n-1} \tilde{S}_{i}+k_{3, n-1} p_{n-1}^{2}+\delta_{n-1},
$$


where all parameters are given in the Appendix as part of the proof of the next proposition. ${ }^{4}$

This value function is similar to that of Foster and Viswanathan (1996) and Holden and Subrahmanyam (1992)..$^{5}$

We now introduce the equilibrium concept used in our model. To start, we define the conditions to be satisfied for a Bayesian Nash equilibrium. Then we restrict our search to linear Markov equilibrium and conjecture the equilibrium strategies for the market maker and informed traders.

Just before period $n$, the information of insider $i$ consists of his own signal $\tilde{S}_{i}$, plus his own orders $\left(\tilde{x}_{i 1}, \ldots, \tilde{x}_{i n-1}\right)$. In addition, all insiders know the past net trades $\left(\tilde{w}_{1}, \ldots, \tilde{w}_{n-1}\right)$. Let

$$
\begin{aligned}
\tilde{x}_{i n} & =X_{i n}\left(\tilde{S}_{i}, \tilde{x}_{i 1}, \ldots, \tilde{x}_{i n-1}, \tilde{w}_{1}, \ldots, \tilde{w}_{n-1}\right) \\
p_{n} & =P_{n}\left(\tilde{w}_{1}, \ldots, \tilde{w}_{n}\right)
\end{aligned}
$$

represent the optimal strategy of trader $i$ and the optimal strategy of the market maker, respectively. Finally, let $X_{i}=\left(X_{i 1}, \ldots, X_{i N}\right)$ (for each $i$ ) and $P=\left(P_{1}, \ldots, P_{N}\right)$ represent the two vectors of strategy functions. Define the profit that accrues to informed trader $i$ from period $n$ on as:

$$
\pi_{n}^{i}\left(X_{1}, \ldots, X_{i}, \ldots, X_{M}, P\right)=\sum_{k=n}^{N}\left(\tilde{v}-p_{k}\right) \tilde{x}_{i k} .
$$

A Bayesian Nash equilibrium of the trading game is a $M+1$ vector of strategies $\left(X_{1}, \ldots, X_{M}, P\right)$ such that (we follow Kyle (1985) and Foster and Viswanathan (1996)

\footnotetext{
${ }^{4}$ We assume here that all insiders play their equilibrium strategies, this expression should be modified if we assume that trader $i$ plays an arbitrary strategy, see appendix for more details.

${ }^{5}$ In Holden and Subrahmanyam (1992), all insiders have the same signal, $\tilde{v}$, implying that the value function of the informed trader $i$ is:

$$
E\left[\pi_{i n} \mid p_{0}, \ldots, p_{i n-1}, \tilde{S}_{i}\right]=\alpha_{n-1}\left(\tilde{v}-p_{n-1}\right)^{2}+\delta_{n-1}
$$

We thus obtain this last value function by setting $k_{1, n-1}=k_{3, n-1}=\alpha_{n-1}$ and $k_{2, n-1}=-2 \alpha_{n-1}$ in our model. Our proof is similar to the one of Foster and Viswanathan (1996) except that we specify the equilibrium conditions for heterogeneously noisy signals.
} 
closely):

- For any $i=1, \ldots, M, n=1, \ldots, N$ and for $X_{i}^{\prime}=\left(X_{i 1}^{\prime}, \ldots, X_{i n}^{\prime}, \ldots, X_{i N}^{\prime}\right)$, we have:

$$
\begin{aligned}
& E\left[\pi_{n}^{i}\left(X_{1}, \ldots, X_{i}, \ldots, X_{M}, P\right) \mid \tilde{S}_{i}, \tilde{x}_{i 1}, \ldots, \tilde{x}_{i n-1}, \tilde{w}_{1}, \ldots, \tilde{w}_{n-1}\right] \\
\geq & E\left[\pi_{n}^{i}\left(X_{1}, \ldots, X_{i}^{\prime}, \ldots, X_{M}, P\right) \mid \tilde{S}_{i}, \tilde{x}_{i 1}, \ldots, \tilde{x}_{i n-1}, \tilde{w}_{1}, \ldots, \tilde{w}_{n-1}\right] .
\end{aligned}
$$

The optimal strategy function for informed trader $i$ is best no matter which past strategies $i$ may have been played.

- For all $n=1, \ldots, N$, we have:

$$
p_{n}=E\left[\tilde{v} \mid \tilde{w}_{1}, \ldots, \tilde{w}_{n}\right]
$$

Also, we define the variance of the price error $\Sigma_{n}$, a measure of price informativeness, at auction $n$ :

$$
\Sigma_{n}=\operatorname{var}\left[\tilde{v} \mid \tilde{w}_{1}, \ldots, \tilde{w}_{n}\right]
$$

The market maker sets prices equal to the conditional expected value given the order flow.

We look for a linear Bayesian Nash equilibrium based on a dynamic programming argument. Note that the strategy of informed trader $i$ at auction $n$ is required to be the optimal strategy, not only when trader $i$ plays his optimal strategy in the first $n-1$ periods. Furthermore, as in Foster and Viswanathan (1996), there are no off equilibrium observations of order flow by the other informed traders in the model as every order flow path is possible.

We now derive the following proposition which provides the different parameters of the equilibrium.

Proposition 1 If $\Sigma_{N}>\frac{\sigma_{\epsilon}^{2}}{M} 6$ there exists a unique linear equilibrium with noisy private information in which the demand function of informed trader $i$ at auction $n$ and the price

\footnotetext{
${ }^{6}$ Expressing equilibrium condition as a function of $\Sigma_{N}$ is equivalent to express the same condition as
} a function of $\Sigma_{0}$. Indeed, we solve our equilibrium by a process of backward induction i.e. we set the 
function at auction $n$ are respectively equal to: ${ }^{7}$

$$
\begin{gathered}
\hat{x}_{i, n}=\alpha_{n} \Delta t \tilde{S}_{i}+\beta_{n} \Delta t \hat{p}_{n-1} . \\
\hat{p}_{n}=\hat{p}_{n-1}+\lambda_{n}\left(\Delta X_{n}+\Delta u_{n}\right),
\end{gathered}
$$

where the parameters are defined by the following equations

$$
\begin{gathered}
\alpha_{n} \Delta t=-\frac{2\left(k_{3 n}-\frac{1}{2} k_{4 n}\right) \lambda_{n}-a_{n}}{(M+1) \lambda_{n}-2 M\left(k_{3 n}-\frac{1}{2} k_{4 n}\right) \lambda_{n}^{2}}+\frac{M-1}{M} \psi_{n}\left(1-a_{n}\right) \frac{1-2 \lambda_{n}\left(k_{3 n}-\frac{1}{2} k_{4 n}\right)}{(M+1) \lambda_{n}-2 M\left(k_{3 n}-\frac{1}{2} k_{4 n}\right) \lambda_{n}^{2}}, \\
\lambda_{n}=\frac{M \alpha_{n} \Delta t \Sigma_{n-1}}{\left(\alpha_{n} \Delta t\right)^{2} M^{2} \Sigma_{n-1}+\sigma_{u}^{2} \Delta t+M\left(\alpha_{n} \Delta t\right)^{2} \sigma_{\epsilon}^{2}} . \\
\Sigma_{n}=\operatorname{var}\left[\tilde{v} \mid \tilde{w}_{1}, \ldots, \tilde{w}_{n}\right]=\frac{\Sigma_{n-1}\left(\sigma_{u}^{2} \Delta t+M\left(\alpha_{n} \Delta t\right)^{2} \sigma_{\epsilon}^{2}\right)}{\left(\alpha_{n} \Delta t\right)^{2} M^{2} \Sigma_{n-1}+\sigma_{u}^{2} \Delta t+M\left(\alpha_{n} \Delta t\right)^{2} \sigma_{\epsilon}^{2}} \\
\text { with } a_{n}=\frac{\Sigma_{n}}{\Sigma_{n}+\left(1-\psi_{n}\right) \sigma_{\varepsilon}^{2}}, \psi_{n}=M \lambda_{n} \alpha_{n} \Delta t a n d \\
\delta_{n-1}=\delta_{n}+\lambda_{n}^{2} k_{3 n}\left[\sigma_{u}^{2} \Delta t+\left(\alpha_{n} \Delta t\right)^{2}(M-1)\left(1+(M-1) a_{n}\right) \sigma_{\epsilon}^{2}\right] .
\end{gathered}
$$

Trader i's value function is given by

$$
E\left[\pi_{i n} \mid \hat{p}_{0}, \ldots, \hat{p}_{n-1}, \tilde{S}_{i}\right]=k_{1, n}\left(\tilde{S}_{i}-\hat{p}_{n-1}\right)+\delta_{n}
$$

The coefficients $k$ 's are solving the following system of equations (fully defined in the Appendix)

$$
k_{n-1}=A k_{n}+C
$$

where $k_{n-1}, k_{n}$ are matrices of dimension $6 \times 1, A$ is a matrix with dimension $6 \times 6$ and $C$ is of dimension $6 \times 1$. All matrices are defined in the Appendix. The parameters are subject to the following boundary conditions

$$
\begin{gathered}
\delta_{N}=k_{1, N}=k_{2, N}=k_{3, N}=0, \\
\alpha_{N} \Delta t=\frac{a_{N}}{\lambda_{N}\left(2+(M-1) a_{N}\right)} .
\end{gathered}
$$

value of $\Sigma_{N}$, then we compute $\Sigma_{N-1} \ldots \Sigma_{0}$. Hence, we can write $\Sigma_{0}$ as a bijective function of $=\Sigma_{N}-$ we observe numerically that $\Sigma_{0}$ is a strictly increasing function of $\Sigma_{N^{-}}$and the equilibrium condition could be interpreted as a condition on $\Sigma_{0}$.

${ }^{7}$ If $\sigma_{\epsilon}^{2}=0$,we are in the Holden and Subrahmanyam (1992) model, the results in this case are presented in the appendix. 


\section{Proof: See Appendix.}

The necessary condition is a learning process condition. It means that the trading process continues as long as informed traders still have some private information that is not yet incorporated in the market maker's information set. It also shows that the precision of the market maker's information is limited by the level of noise contained in the traders' signals. In fact, we know that the market makers' estimate of the liquidation value can be written as:

$$
\hat{v}=\frac{1}{M} \sum_{i=1}^{M} \tilde{S}_{i}=\tilde{v}+\frac{1}{M} \sum_{i=1}^{M} \tilde{\epsilon}_{i} .
$$

Suppose that the traders' signals $\left(\tilde{S}_{i}\right)_{1 \leq i \leq M}$ are in the information set of the market maker. In this case, the market maker is able to know the liquidation value $\tilde{v}$ with a precision measured by the inverse of the variance of the random variable $\frac{1}{M} \sum_{i=1}^{M} \tilde{\epsilon}_{i}$. That precision is then equal to $\frac{\sigma_{\epsilon}^{2}}{M}$ and represents the best precision of her estimate of the liquidation value $\tilde{v}$. The last error variance of price, $\Sigma_{N}$, is then greater than $\frac{\sigma_{\varepsilon}^{2}}{M}$. As a consequence, the level of noise, as measured by the variance of the noise $\sigma_{\varepsilon}^{2}$ cannot be too high.

Moreover, we find, numerically, that if we increase the frequency of trading $N$, we need to set lower values of $\Sigma_{N}$ to maintain the same $\Sigma_{0} \cdot{ }^{8}$ That allows us to deduce that the frequency of trading is limited by the level of noise. The higher the frequency of trading, the lower the level of noise. Regarding the effect of the number of insiders $M$, it is not as clear. Increasing the number of insiders $M$ decreases the lower bound of the necessary condition. However, it also intensifies the competition between traders and so lead to lower values of $\Sigma_{N}$. Hence, we cannot deduct analytically the effect of increasing the number of informed traders.

By proceeding by backward induction one determines the individual orders for each auction. There is then a link between the last error variance of price $\Sigma_{N}$ and the initial one $\Sigma_{0}$ at the opening of the sequential auctions market. Choosing $\Sigma_{0}$ is therefore equivalent

\footnotetext{
${ }^{8}$ This is due to the fact that increasing the frequency of trading intensifies the competition between traders leading to more information being released and so to lower values of $\Sigma_{N}$.
} 
to setting $\Sigma_{N}$ at a certain value. To illustrate the properties of our model, we compute the linear equilibrium parameters for different settings.

All the results in the following sections are obtained numerically.

\section{$4 \quad$ Numerical Results}

We now illustrate our model with numerical simulations. In order to compare the different results we choose similar numerical settings to those of Holden and Subrahmanyam (1992), Foster and Viswanathan (1996) and Back et al. (2000). The results are simulated for a fixed initial value of $\Sigma_{0}$.

\subsection{Informativeness and liquidity}

We are interested in how prices aggregate the different pieces of private information held by informed traders. In the next result, we study the informativeness of prices.

Numerical result 1 When the level of noise in the traders' signals is low, prices incorporate quickly all the traders' private information. When the signals become noisier the decay of $\Sigma_{n}$ becomes slower and less information is acquired by the market maker during trading.

In our model, the conditional correlation between the signals of the informed market participants cannot be negative. As a consequence, traders trade on the same side of the market. Nevertheless, that competition is softened as traders have noisy signals. We can compare our model to that of Foster and Viswanathan (1996) by looking at the correlation between the signals. The correlation between the informed agents' private signals, $i$ and $j$ at time $n$, is given by:

$$
\operatorname{corr}\left(\tilde{S}_{i}, \tilde{S}_{j}\right)_{n}=\frac{\Sigma_{n}}{\Sigma_{n}+\sigma_{\epsilon}^{2}} \quad \text { for } \quad i \neq j
$$

It can be seen from this expression that the value of $\sigma_{\epsilon}^{2}$ impacts the correlation between two signals. This correlation affects the traders' behavior, which in turn impacts price 
informativeness. However, the variance of the noise also affects the traders' behavior independently from its effect on the correlation. Noisier (more precise) signals also implies that informed agents, ceteris paribus, trade more (less) on noise reducing (increasing) price informativeness.

In Figures 1 and 2, we represent the error variance of prices over time for different values of the correlation between the signals of the informed traders (given by $\sigma_{\epsilon}^{2}$ ).

Figure 1: The error variance of prices $\left(\Sigma_{n}\right) \quad$ Figure 2: The error variance of prices $\left(\Sigma_{n}\right)$ over time, $M=2, N=4, \sigma_{u}^{2}=1, \Sigma_{0}=1 . \quad$ over time, $M=2, N=50, \sigma_{u}^{2}=1, \Sigma_{0}=1$.

Figure 1 shows that the error variance of price $\Sigma_{n}$ decreases more slowly when the variance of the noise, $\sigma_{\epsilon}^{2}$, is large. In other words, the noisier the signal the less information is revealed during the periods of trading. Figure 1 also shows that, when the trader's private information is not too noisy, investors reveal more of their private information in the early auctions whereas the opposite is true when private information is very noisy. These effects can be explained as follows: when the level of noise is low, traders compete more aggressively on their private information and so release the largest part of their information in the early periods of trading, but when the level of noise is high, each trader prefers to delay his trades and keeps most of his private information to the last auctions. These effects are shown numerically in Figure 2.

In the next result, we study the liquidity in the market. 
Numerical result 2 The liquidity parameter $\lambda_{n}$ is non monotonic with $\sigma_{\epsilon}^{2}$. It decreases over time when the level of noise is low. When the level of noise is large, it increases over time.

Figure 3: The liquidity parameter $\left(\lambda_{n}\right)$ over

Figure 4: The liquidity parameter $\left(\lambda_{n}\right)$ over time, $M=2, N=4, \sigma_{u}^{2}=1, \Sigma_{0}=1$. time, $M=2, N=50, \sigma_{u}^{2}=1, \Sigma_{0}=1$.

Figure 3 displays the dynamic of the liquidity parameter as a function of the noise. It shows that as more information is incorporated into prices, the less aggressively the market maker prices the asset. It also shows that, when there is a large level of noise in the private signals, the market maker's sensitivity to order flow, $\lambda_{n}$, increases slowly through time. This is due to the fact that, in that case, informed traders delay their trades to the last auctions and do not reveal a large part of their private information. Hence, the market maker cannot learn much about the liquidation value of the asset in the early periods of trading and she reacts more aggressively to the order flow that appears in the last periods of trading. These results are shown numerically in Figure 4.

We now focus on the link between the informativeness of prices and the number of auctions. Figure 5 shows the informativeness of prices for different values of the number of auctions. In order to guarantee the existence of an equilibrium for the different parameter configurations, we take $\sigma_{\epsilon}^{2}=0.02$. 
Hence, one can observe that for a fixed level of noise, $\sigma_{\epsilon}^{2}$, the informativeness of prices increases with the number of auctions.

Figure 5: The error variance of prices $\left(\Sigma_{n}\right)$

Figure 6: The liquidity parameter $\left(\lambda_{n}\right)$ for

for different values of $N, M=2, \sigma_{\epsilon}^{2}=0.02$, different values of $N, M=2, \sigma_{\epsilon}^{2}=0.02, \sigma_{u}^{2}=$ $\sigma_{u}^{2}=1, \Sigma_{0}=1$. $1, \Sigma_{0}=1$.

Similarly, Figure 6 shows that the adverse selection problem (measured by the parameter $\lambda_{n}$ ) decreases with the number of auctions as one approaches the end of the trading day. It also shows that the larger the number of auctions, the higher the price is at the first auction.

Figures 7 and 8 show the links between the informativeness of prices and the number of traders, and between the liquidity and the number of traders. One can see that for a fixed level of noise, the informativeness of prices increases with the number of traders.

Also, we observe from Figure 8 that the liquidity parameter $\lambda_{n}$ decreases with the number of traders. Then, we conclude that increasing the number of traders or auctions boosts the competition between traders and so leads to the release of more information to the market maker.

We now study the reaction of the traders to their private and public information, and present the different regimes of competition between traders. 
Figure 7: The error variance of prices $\left(\Sigma_{n}\right)$

for different values of $M, N=4, \sigma_{\epsilon}^{2}=0.02$, $\sigma_{u}^{2}=1, \Sigma_{0}=1$.
Figure 8: The liquidity parameter $\left(\lambda_{n}\right)$ for different values of $M, N=4, \sigma_{\epsilon}^{2}=0.02, \sigma_{u}^{2}=$ $1, \Sigma_{0}=1$.

\subsection{Competition: The rat race and the waiting game}

We are interested in how noise affects the competition between informed traders. In the next result, we study the reaction of informed traders to their public and private information.

Numerical result 3 The informed market participants react more to both their private and public information as time elapses. When the level of noise is low, the informed traders react aggressively to their private and public information and increase significantly their orders in the last periods of trading. The reaction of informed traders becomes less agressive when the level of noise increases. Hence, when the level of noise is large, informed traders react less aggressively to their information during all the periods of trading.

These results are shown in Figures 9 and 10.

Figures 9 and 10 show that the informed traders trade gradually more aggressively on both their private and public information. It can be seen that the more precise the signal the more aggressive the traders are. This aggressive trading is what we call a rat race. When the level of noise is low, we observe a rat race during all the periods of trading. 
Figure 9: The reaction of an informed investor to his private signal $\left(\alpha_{n}\right)$ over time, $M=2, N=4, \sigma_{u}^{2}=1, \Sigma_{0}=1$.
Figure 10: The reaction of an informed investor to the prices $\left(\beta_{n}\right)$ over time, $M=2$, $N=4, \sigma_{u}^{2}=1, \Sigma_{0}=1$.

As the level of noise increases, the intensity of this rat race decreases. In the presence of high noise, traders are not aggressive in their trading during the early periods of trading, we call that a waiting game. However, we still have a rat race in the last periods of trading. Regarding the traders' behavior with respect to their private information, this can be explained as follows. Firstly, as time gets closer to the end of the trading day informed traders have less scope to use their private information. Secondly, the impact of their trades has less long lasting effect on the liquidity. The intensity of the traders' trading decreases with $\sigma_{\varepsilon}^{2}$ (this also includes the traders' behavior at the late auctions). As said before, in the early auctions we observe that traders are not comparatively aggressive in their trading. As the noise in their information is not too high, traders refrain from trading too early as trading aggressively would lead to their private information being incorporated in the price early. However, when the level of noise is very high, we only observe a waiting game. In this case, the high level of noise present in the traders' signals reduces the competition between informed traders since expression (4.1) is close to zero in that case. Indeed, when $\sigma_{\varepsilon}^{2}$ is high, traders have initially more dispersed information reducing the correlation between the traders signals. This in turn limits the competition 
between traders during all the periods of trading as this conditional correlation remains low.

The waiting game observed in our model is not due to a negative correlation between the signals as a consequence of trading. We show that, in our model, the waiting game phase appears when the correlation between the signals of the traders is low - but positive. In our model, increasing the level of noise in the traders signals decreases the correlation between these signals. Then, we have for a high level of noise:

$$
\operatorname{Corr}\left(\tilde{S}_{i}, \tilde{S}_{j}\right)_{n} \approx 0
$$

Hence, in this case, each trader considers that the information from other traders is completely uncorrelated to the true value of the asset. Therefore, he limits his orders during the early periods of trading in order to not reveal his private information and waits for the last periods to submit more significant orders. ${ }^{9}$

Figures 11 and 12 show that, for low levels of noise, we only observe a rat race during all the periods of trading: we can see from these figures that the traders' reaction to their information increases rapidly during all the periods of trading. We also observe that the slope of the parameter $\alpha_{n}$ (which measures the intensity of competition) increases during all the periods of trading and more significantly in the lasts periods. This result generalizes the findings of Kyle (1985) and Holden and Subrahmanyam (1992) in the case of signals with low levels of noise.

Figures 11 and 12 also show that, for intermediate levels of noise, we observe a waiting game that lasts most of the auctions as the trading intensity dramatically increases in the last auctions. The Figures also show that the intensity of that rat race decreases with the level of noise. Hence, for very high levels of noise, we only observe a waiting game that lasts for all the periods of trading. ${ }^{10}$ The main difference with Foster and Viswanathan

\footnotetext{
${ }^{9}$ In this case, we can compare our model to the one of Kyle (1985), since each informed trader considers other traders as noise traders, and so follows a strategy comparable to that observed in Kyle (1985).

${ }^{10}$ In fact, we always observe a rat race at the last auctions. However, the intensity of this rat race decreases with the level of noise and becomes difficult to observe when the level of noise is too high.
} 
(1996) is the order in which the two game stages can appear.

Figure 11: Reaction of an informed investor to his private signal $\left(\alpha_{n}\right)$ over time, $M=2$, $N=20, \sigma_{u}^{2}=1, \Sigma_{0}=1$.
Figure 12: Reaction of an informed investor to his private signal $\left(\alpha_{n}\right)$ over time, $M=2$, $N=100, \sigma_{u}^{2}=1, \Sigma_{0}=1$.

When we analyze the effect of increasing the number of auctions on the previous result, we obtain the following results. As an overall, increasing the number of auctions increases the traders' aggressiveness in each auction. For a very low level of noise and when increasing the number of auctions, we still have a rat race during all the periods of trading. ${ }^{11}$ We also observe that the intensity of the rat race increases with the number of auctions. ${ }^{12}$ When increasing the number of auctions for a high level of noise, a waiting game takes place for most of the auctions. We also obtain that increasing the number of auctions increases the intensity of the rat race observed in the last auctions.

When we analyze the effect of increasing the number of insiders on the previous result, we obtain the following results. For a very low level of noise, we still have one phase only, i.e. the rat race. The results show that the intensity of this rat race increases with the

\footnotetext{
${ }^{11}$ The simulations show that the range of $\sigma_{\epsilon}^{2}$ for which this result is satisfied becomes smaller and closer to 0 when the number of periods increases.

${ }^{12}$ Numerically, we observe higher final values of $\alpha_{n}$ when we increase the number of auctions $N$, and keep $\sigma_{\epsilon}$ constant.
} 
Figure 13: Reaction of an informed investor to his private signal $\left(\alpha_{n}\right)$ over time, $M=10$, $N=20, \sigma_{u}^{2}=1, \Sigma_{0}=1$.
Figure 14: Reaction of an informed investor to his private signal $\left(\alpha_{n}\right)$ over time, $M=10$, $N=100, \sigma_{u}^{2}=1, \Sigma_{0}=1$.

number of insiders. ${ }^{13}$ We obtain that, for high levels of noise, the competition is distorted as follows: we have an early and a late rat race with a waiting game occurring between those two rat races. The intensity of the early rate race increases with the number of insiders as well as with the number of auctions. It also increases when signals become more precise (see Figures 13 and 14). The intensity of the final rat race decreases with the number of traders.

\subsection{Profit}

In this section, we are interested in understanding how the competition between the insiders affects their profits.

Numerical result 4 1. The introduction of the noise in the traders' signals reduces the profits when the level of the overall competition is low, measured by both the temporal and the spatial competition i.e. when $M=2$ and $N<7$, or $M=3$ and $N<3$, or $M=4$ and $N=1$.

\footnotetext{
${ }^{13}$ These results are obtained for a range of very low levels of noise, this range gets narrower when the number of insiders increases.
} 
2. When the level of competition is high ( $M=2$ and $N \geq 7$, or $M=3$ and $N \geq 3$ or $M=4$ and $N \geq 2$, or $M \geq 5$ and for any $N)$, the traders' profits are non-monotonic with the level of noise. More precisely, the profits intially increase with low level of noise and then decrease with it for high value of the noise.

Figure 15: The individual profit $\left(\pi_{i}\right)$ as a

Figure 16: The individual profit $\left(\pi_{i}\right)$ as a function of $\sigma_{\varepsilon}^{2}$ for $M=2$, the number of function of $\sigma_{\varepsilon}^{2}$ for $M=4$, the number of insiders, $N=3$, the number of auctions, insiders, $N=10$, the number of auctions, $\sigma_{u}^{2}=1, \Sigma_{0}=1 . \quad \sigma_{u}^{2}=1, \Sigma_{0}=1$.

These results can be explained as follows. Introducing noise in the traders' signals diminishes the intensity of the competition and so allows the traders to get greater profits (Figure 16). However, too much noise decreases the trader's trading intensity in such a way that traders switch to a waiting game and so diminishes the profits. Noise acts as a commitment not to trade. When the competition is low (measured by both $N$ and $M$ ), only the negative effect of the noise is present (Figure 15).

\subsection{Optimal noise}

In this section, we look at the optimal level of noise, $\sigma_{\epsilon}^{2}$ maximizing the informed traders' expected profit. Our previous results show that the presence of noise in the private signals 
may reduce the level of competition between informed traders. This would lead to higher expected profits.

Numerical result 5 There exists an optimal level of noise which maximizes the expected profits of the traders when the number of insiders is relatively high. We show numerically that this level increases with the number of insiders, that the optimal individual profit decreases with $M$ and that the optimal aggregate profit converges to a finite positive value when $M$ increases infinitely.

These results are shown in Figures 17, 18 and 19.

Figure 17: The individual profit $\left(\pi_{i}\right)$ as a function of $\sigma_{\varepsilon}^{2}$ for $N=4$, the number of auctions, $\sigma_{u}^{2}=1, \Sigma_{0}=1$.
Figure 18: The individual profit $\left(\pi_{i}\right)$ as a function of $\sigma_{\varepsilon}^{2}$ for $N=4$, the number of auctions, $\sigma_{u}^{2}=1, \Sigma_{0}=1$.

Figures 17 and 18 show the individual profit for different values of the number of insiders. We obtain that the optimal level of noise, i.e. the level of noise maximizing the traders' expected profit, increases with the number of auctions. We also observe that the optimal individual profit, computed as the profit obtained from the first auction to the last evaluated at the optimal amount of noise, decreases with the number of insiders. This result is similar to the one obtained by Dridi and Germain (2009). 
Figure 19: The aggregate profit $\left(\pi_{A g g}\right)$ as a function of $\sigma_{\varepsilon}^{2}$ for $N=4$, the number of auctions, $\sigma_{u}^{2}=1, \Sigma_{0}=1$.
Figure 20: The individual profit $\left(\pi_{i}\right)$ as a function of $\sigma_{\varepsilon}^{2}$ for $M=2$, the number of insiders, $\sigma_{u}^{2}=1, \Sigma_{0}=1$.

Figure 19 shows that the expected aggregate profit (individual profit aggregated over the number of traders) does not go to zero when $M$ is large, but instead tends to a finite positive value. This result is similar to the one obtained by Dridi and Germain (2009).

The next result exhibits the effects of increasing the number of auctions on the traders expected profits.

Numerical result 6 The optimal level of noise increases with the number of auctions. ${ }^{14}$ For a fixed level of noise, the individual profits decrease with the number of auctions $N$ for low levels of noise, whereas it increases with the number of auctions for high levels of noise. We also obtain that the optimal individual profit converges to a finite positive value when $N$ increases infinitely.

These results are shown numerically in Figure 20.

The previous result can be explained as follows: as we increase the number of auctions, the informed traders scope of profit increases. However, when the level of noise is low, the profit decreases with the number of auctions since, in this case, the traders' private

\footnotetext{
${ }^{14}$ We observe numerically that the optimal level of noise increases with $N$ slower than with $M$.
} 
information is quickly incorporated in the price which then converges quickly to the true value of the asset. When the level of noise is relatively high, less information is revealed to the market maker and so the price does not converge as quickly to the true value of the asset allowing the traders to obtain larger profits.

\section{Conclusion}

This article analyzes the introduction of heterogeneous noisy signals when strategic insiders compete in a multi-auction market. In this case, when all informed agents are endowed with signals of the same precision, we derive the unique linear equilibrium and its properties. We find that the existence of an equilibrium is not always guaranteed. The existence condition implies a negative relationship between the number of auctions and the noise in the traders' private signal. The existence of the equilibrium is guaranteed when the competition is limited through noisy signals.

Our model also enables us to analyze the trade-off between noise and competition as in

Dridi and Germain (2009). We show that when the competition is strong (the number of informed traders and/or the number of auctions is high), noisy information can reduce the competition between insiders and can increase their expected profits. In that case noise acts as a commitment not to trade.In the case of an intense competition, a low level of noise reduces the competition between traders and leads to greater profits. For a weak competition (the number of informed traders and the number of auctions are low), the introduction of some noise in the traders' signals always leads to a drop in their expected profits. We then obtain a result which is similar to Dridi and Germain (2009).

With an intense competition, there exists an optimal level of noise that maximizes the expected profits of the traders. We obtain that this level increases indefinitely with the number of traders and the number of auctions. We also obtain, as in Dridi and Germain (2009), that the optimal aggregate profit has a strictly positive finite limit when the number of traders is large. Moreover, the optimal individual profit has a strictly positive finite limit 
when the number of auctions is large.

Furthermore, when the level of the noise is small leading to a strong correlation between signals, the competition between traders takes the form of a rat race during all the periods of trading. However, as we increase the level of noise, a waiting game phase appears during

the early periods of trading, and the intensity of the rat race of the last auctions decreases. Hence, when the level of the noise is too large (implying that the correlation is weak) we only observe a waiting game. This result is in sharp contrast with Foster and Viswanathan (1996).

Our paper broadly agrees with the findings of Kyle (1985) where the traders gradually incorporates their information into price. We find that traders trade very aggressively at the last auction, as in Kyle (1985), however we do observe an auction where they refrain from trading and even decrease their trading intensity. One empirical prediction of this model is that changes in volume during the trading day can be explained by the presence of noise in the information of the traders when they compete in the market. It would be difficult to test this model empirically as we don't have access to the profit and the information of traders in banks. Nevertheless, we could design an experiment where the level of noise is controlled and the profit of the players measured during the trading game.

\section{Appendix}

\section{Proof of Proposition 1}

The proof involves four steps. We will start by resolving the dimensionality issue (we avoid the problem of increasing state history with time) when all traders follow their optimal strategies. In the second step, we resolve the dimensionality issue when one trader deviates from his optimal strategy, conjecture the value function and then obtain the first order condition (FOC) that determines the equilibrium. In the third step, we show with a lemma that, at the equilibrium, the parameters of the demand function for insider $i$ do not depend on $i$. In other words, at the equilibrium, all insiders have the same reaction to 
their private information $\left(\alpha_{n}\right)$ and to their public information $\left(\beta_{n}\right)$. Finally, in the fourth step, we derive the insiders' backward induction program.

\section{Step 1: The Dimensionality Issue}

In this section we show how the dimensionality issue is resolved (i.e. we avoid the problem of increasing state history with time). As in Foster and Viswanathan (1996), we look at linear strategies for informed traders and learning by the market maker.

Consider trader $i$ who is interested in forecasting the true value of the asset that is not predicted by the market after $n-1$ periods of trading, using his information $\left(\tilde{S}_{i}, \tilde{x}_{i 1}, \ldots, \tilde{x}_{i n-1}, \tilde{w}_{1}, \ldots, \tilde{w}_{n-1}\right)$. By equation (3.4), it can be shown that:

$$
p_{n}=p_{0}+\sum_{k=1}^{n} \lambda_{k} \tilde{w}_{k}
$$

Trader $i$ 's order for $r=1, \ldots, n-1$ can be rewritten as:

$$
\tilde{x}_{i r}=\alpha_{i r} \Delta t_{r} \tilde{S}_{i}+\beta_{r} \Delta t_{r} p_{r-1}=\alpha_{i r} \Delta t_{r} \tilde{S}_{i}+\beta_{r} \Delta t_{r}\left(p_{0}+\sum_{k=1}^{r-1} \lambda_{k} \tilde{w}_{k}\right) .
$$

It is then clear that $\left(\tilde{x}_{i 1}, \ldots, \tilde{x}_{i n-1}\right)$ is redundant and the meaningful history for trader $i$ is just $\left(\tilde{S}_{i}, \tilde{w}_{1}, \ldots, \tilde{w}_{n-1}\right)$. It is important to note that this only holds when trader $i$ plays his conjectured optimal strategy in past periods. In developing this result, we exploit the fact that optimal strategies are functions of the private signal $\tilde{S}_{i}$, and the order flow up to that point. Thus trader $i$ predicts $\tilde{v}-p_{n-1}$ as follows:

$$
\begin{aligned}
E\left[\tilde{v}-p_{n-1} \mid \tilde{S}_{i}, \tilde{w}_{1}, \ldots, \tilde{w}_{n-1}, \tilde{x}_{i 1}, \ldots, \tilde{x}_{i n-1}\right] & =E\left[\tilde{v}-p_{n-1} \mid \tilde{S}_{i}, \tilde{w}_{1}, \ldots, \tilde{w}_{n-1}\right] \\
& =E\left[\tilde{v}-p_{n-1} \mid \tilde{S}_{i}-p_{n-1}, \tilde{w}_{1}, \ldots, \tilde{w}_{n-1}\right] \\
& =E\left[\tilde{v}-p_{n-1} \mid \tilde{S}_{i}-p_{n-1}\right] \\
& =a_{n}\left(\tilde{S}_{i}-p_{n-1}\right) .
\end{aligned}
$$

We can then deduce that $\tilde{S}_{i}-p_{n-1}$ is a sufficient statistic for trader $i$ to predict the value of the asset after $n-1$ trading periods. In deriving this result, we first use the fact that in equilibrium $\left(\tilde{x}_{i 1}, \ldots, \tilde{x}_{i n-1}\right)$ is redundant given $\left(\tilde{S}_{i}, \tilde{w}_{1}, \ldots, \tilde{w}_{n-1}\right)$. Then, we use the result that when we project $\tilde{v}$ and $\tilde{S}_{i}$ on $\left(\tilde{w}_{1}, \ldots, \tilde{w}_{n-1}\right)$ we obtain $p_{n-1}$, so both 
$\tilde{v}-p_{n-1}$ and $\tilde{S}_{i}-p_{n-1}$ are independent of $\left(\tilde{w}_{1}, \ldots, \tilde{w}_{n-1}\right)$. Trader $i$ is also interested in predicting the signals of the other informed traders, as they use their signals to submit orders, which in turn determine market price. Because trader $j$ submits an order of the form $\tilde{x}_{j n}=\alpha_{j n} \Delta t_{n} \tilde{S}_{j}+\beta_{n} \Delta t p_{n-1}$, trader $i$ needs to predict $\tilde{S}_{j}$. This is done as follows:

$$
\begin{aligned}
E\left[\tilde{S}_{j} \mid \tilde{S}_{i}, \tilde{w}_{1}, \ldots, \tilde{w}_{n-1}\right] & =E\left[\tilde{S}_{j}-p_{n-1} \mid \tilde{S}_{i}, \tilde{w}_{1}, \ldots, \tilde{w}_{n-1}\right]+p_{n-1} \\
& =E\left[\tilde{S}_{j}-p_{n-1} \mid \tilde{S}_{i}-p_{n-1}, \tilde{w}_{1}, \ldots, \tilde{w}_{n-1}\right]+p_{n-1} \\
& =E\left[\tilde{S}_{j}-p_{n-1} \mid \tilde{S}_{i}-p_{n-1}\right]+p_{n-1} \\
& =E\left[\tilde{v}+\tilde{\epsilon}_{j}-p_{n-1} \mid \tilde{S}_{i}-p_{n-1}\right]+p_{n-1} \\
& =E\left[\tilde{v}-p_{n-1} \mid \tilde{S}_{i}-p_{n-1}\right]+p_{n-1} \\
& =a_{n}\left(\tilde{S}_{i}-p_{n-1}\right)+p_{n-1} .
\end{aligned}
$$

So $\left(\tilde{S}_{i}-p_{n-1}, p_{n-1}\right)$ is a sufficient statistic for trader $i$ to predict $\tilde{S}_{j}$, and there is no historydependent hierarchy of forecasts. The above discussion shows how the dimensionality issue is resolved in our model when all traders submit their optimal orders. However, we must also consider deviations from the optimal strategy by any one trader (keeping the behavior of other traders fixed). If trader $i$ submits an arbitrary order sequence $\left(\tilde{x}_{i 1}, \ldots, \tilde{x}_{i n-1}\right)$, which is different from the equilibrium orders (given by equation (3.3)), the sufficient statistics that we have computed need not be relevant. ${ }^{15}$ In the next step, we resolve the dimensionality issue when one trader deviates from his optimal strategy (keeping the strategies of other traders fixed) and find the necessary and sufficient conditions for the equilibrium. This finishes step 1 of the proof.

\section{Step 2: Necessary and Sufficient Conditions For Equilibrium.}

Suppose that all traders other than trader $i$ play their conjectured equilibrium strategy and the market maker updates her beliefs using the linear rules described above. Now consider what happens if trader $i$ has submitted arbitrary orders in the first $n-1$ periods $\left(\tilde{x}_{i 1}, \ldots, \tilde{x}_{i n-1}\right)$. To solve the model in this setting, we first need to construct the following

\footnotetext{
${ }^{15}$ In particular $\tilde{S}_{i}-p_{n-1}$ is not orthogonal to $\left(\tilde{w}_{1}, \ldots, \tilde{w}_{n-1}\right)$ since $p_{n-1} \neq E\left[\tilde{v} \mid \tilde{w}_{1}, \ldots, \tilde{w}_{n-1}\right]$ because trader $i$ has not played his optimal strategy in the first $n-1$ rounds of trading.
} 
statistics based on trader $i$ 's information from the first $n-1$ periods. These statistics correspond to the outcomes that would have occurred had trader $i$ used the equilibrium strategy instead of the arbitrary strategy in the first $n-1$ periods. For each informed trader, we recursively define:

$$
\begin{gathered}
\hat{w}_{n}^{i}=\sum_{j=1}^{M}\left(\alpha_{j n} \Delta t \tilde{S}_{j}+\beta_{n} \Delta t \hat{p}_{n-1}^{i}\right)+\Delta u_{n}, \\
\hat{p}_{n}^{i}=p_{0}+\sum_{k=1}^{n} \lambda_{k} \hat{w}_{k}^{i},
\end{gathered}
$$

where $\hat{w}_{n}^{i}$ is the order flow that would have occurred in the $n^{\text {th }}$ round of trading if trader $i$ had followed the equilibrium strategy $\left(\hat{x}_{i 0}, \ldots, \hat{x}_{i n-1}\right)$ in the first $n$ periods of trading. Similarly, after $n$ rounds of trading, $\hat{p}_{n}^{i}$ is the price that prevails in the $n^{\text {th }}$ round of trading if trader $i$ had followed the equilibrium strategy $\left(\hat{x}_{i 0}, \ldots, \hat{x}_{i n-1}\right)$ in the first $n$ periods of trading.

We can prove by mathematical induction that $\left(\hat{w}_{k}^{i}, \hat{p}_{k}^{i}\right)$ is in the information set of trader $i$ $\left(\tilde{S}_{i}, \tilde{w}_{1}, \ldots, \tilde{w}_{n-1}, \tilde{x}_{i 1}, \ldots, \tilde{x}_{i n-1}\right)$. Hence, trader $i$ knows the change in the order flow and the market maker's expectation of the liquidation value of the asset.

As in Foster and Viswanathan (1996), the price deviation from equilibrium caused by past suboptimal play is the additional variable that is needed to summarize the history observed by trader $i$ :

$$
\begin{aligned}
E\left[\tilde{v}-p_{n-1} \mid \tilde{S}_{i}, \tilde{w}_{1}, \ldots, \tilde{w}_{n-1}, \tilde{x}_{i 1}, \ldots, \tilde{x}_{i n-1}\right] & =E\left[\tilde{v}-\hat{p}_{n-1}^{i}+\hat{p}_{n-1}^{i}-p_{n-1} \mid \tilde{S}_{i}\right. \\
\left.\tilde{w}_{1}, \ldots, \tilde{w}_{n-1}, \tilde{x}_{i 1}, \ldots, \tilde{x}_{i n-1}\right] & =E\left[\tilde{v}-\hat{p}_{n-1}^{i} \mid \tilde{S}_{i}, \tilde{w}_{1}, \ldots, \tilde{w}_{n-1}, \tilde{x}_{i 1}, \ldots, \tilde{x}_{i n-1}\right] \\
+\hat{p}_{n-1}^{i}-p_{n-1} & =E\left[\tilde{v}-\hat{p}_{n-1}^{i} \mid \tilde{S}_{i}, \hat{w}_{1}^{i}, \ldots, \hat{w}_{n-1}^{i}, \tilde{x}_{i 1}, \ldots, \tilde{x}_{i n-1}\right]+\hat{p}_{n-1}^{i}-p_{n-1} \\
& =a_{n}\left(\tilde{S}_{i}-\hat{p}_{n-1}^{i}\right)+\hat{p}_{n-1}^{i}-p_{n-1}
\end{aligned}
$$

Here, we use the fact that $\hat{p}_{n-1}^{i}-p_{n-1}$ is in the information set of trader $i$ and that $\left(\tilde{S}_{i}, \tilde{w}_{1}, \ldots, \tilde{w}_{n-1}, \tilde{x}_{i 1}, \ldots, \tilde{x}_{i n-1}\right)$ can be constructed from $\left(\tilde{S}_{i}, \hat{w}_{1}^{i}, \ldots, \hat{w}_{n-1}^{i}, \tilde{x}_{i 1}, \ldots, \tilde{x}_{i n-1}\right)$. 
We also have:

$$
\begin{aligned}
E\left[\tilde{S}_{j} \mid \tilde{S}_{i}, \tilde{w}_{1}, \ldots, \tilde{w}_{n-1}, \tilde{x}_{i 1}, \ldots, \tilde{x}_{i n-1}\right] & =E\left[\tilde{S}_{j}-\hat{p}_{n-1} \mid \tilde{S}_{i}, \tilde{w}_{1}, \ldots, \tilde{w}_{n-1}\right. \\
\left.\tilde{x}_{i 1}, \ldots, \tilde{x}_{i n-1}\right]+\hat{p}_{n-1} & =a_{n}\left(\tilde{S}_{i}-\hat{p}_{n-1}^{i}\right)+\hat{p}_{n-1}^{i}
\end{aligned}
$$

Hence, we find that $\left(\tilde{S}_{i}-\hat{p}_{n-1}^{i}, \hat{p}_{n-1}^{i}-p_{n-1}, \hat{p}_{n-1}^{i}\right)$ is a sufficient statistic to forecast the liquidation value and the signals of other traders. Now, we conjecture the value function of trader $i$ after $n-1$ to be:

$$
\begin{aligned}
\pi_{i n}= & k_{1, n-1} \tilde{S}_{i}^{2}+k_{2, n-1} \tilde{S}_{i} p_{n-1}+k_{3, n-1} p_{n-1}^{2}+k_{4, n-1} p_{n-1}\left(\hat{p}_{n-1}^{i}-p_{n-1}\right) \\
& +k_{5, n-1} \tilde{S}_{i}\left(\hat{p}_{n-1}^{i}-p_{n-1}\right)+k_{6, n-1}\left(\hat{p}_{n-1}^{i}-p_{n-1}\right)^{2}+\delta_{n-1} .
\end{aligned}
$$

We also conjecture the optimal strategy of a trader who has played an arbitrary strategy:

$$
\tilde{x}_{i k}=\alpha_{i k} \Delta t_{k} \tilde{S}_{i}+\beta_{k} \Delta t_{k} p_{k-1}+\zeta_{k} \Delta t_{k}\left(\hat{p}_{k-1}^{i}-p_{k-1}\right) .
$$

One can consider the profit which is realized at the $n t h$ auction, and what remains to be gained from the next auction to the end of trading. This is given below:

$E\left[\pi_{i n} \mid \tilde{S}_{i}, \tilde{w}_{1}, \ldots, \tilde{w}_{n-1}, \tilde{x}_{i 1}, \ldots, \tilde{x}_{i n-1}\right]=E\left[\left(\tilde{v}-p_{n}\right) \tilde{x}_{i n}+\pi_{n+1} \mid \tilde{S}_{i}, \tilde{w}_{1}, \ldots, \tilde{w}_{n-1}, \tilde{x}_{i 1}, \ldots, \tilde{x}_{i n-1}\right]$

This leads to the following expression

$$
E\left[\pi_{i n} \mid \tilde{S}_{i}, \tilde{w}_{1}, \ldots, \tilde{w}_{n-1}, \tilde{x}_{i 1}, \ldots, \tilde{x}_{i n-1}\right]=I+I I,
$$

with

$$
\begin{gathered}
I=E\left[\tilde{x}_{i n}\left(\tilde{v}-p_{n}\right) \mid \tilde{S}_{i}, \tilde{w}_{1}, \ldots, \tilde{w}_{n-1}, \tilde{x}_{i 1}, \ldots, \tilde{x}_{i n-1}\right], \\
I I=E\left[\pi_{i n+1} \mid \tilde{S}_{i}, \tilde{w}_{1}, \ldots, \tilde{w}_{n-1}, \tilde{x}_{i 1}, \ldots, \tilde{x}_{i n-1}\right] .
\end{gathered}
$$

The price at auction $n$ is given by

$$
p_{n}=p_{n-1}+\lambda_{n}\left(\Delta \tilde{X}_{n}+\Delta \tilde{u}_{n}\right)
$$

with $\Delta \tilde{X}_{n}=\tilde{x}_{i n}+\Delta X_{n}^{*}$ the aggregate order flow from the demand of the $i t h$ insider $\left(\tilde{x}_{i n}\right)$ and from the $M-1$ other informed participants $\left(\Delta X_{n}^{*}\right)$ at the $n t h$ auction. 
Since $\Delta \tilde{u}_{n}$ is independent of $\tilde{v}$, we obtain

$$
E\left[\Delta \tilde{u}_{n} \mid p_{1}, \ldots, p_{n-1}, \tilde{S}_{i}\right]=E\left[\Delta \tilde{u}_{n}\right]=0
$$

We have already shown that:

$$
E\left[\widetilde{v} \mid \tilde{S}_{i}, \tilde{w}_{1}, \ldots, \tilde{w}_{n-1}, \tilde{x}_{i 1}, \ldots, \tilde{x}_{i n-1}\right]=a_{n}\left(\tilde{S}_{i}-\hat{p}_{n-1}^{i}\right)+\hat{p}_{n-1}^{i} .
$$

Due to normality, we have the standard formula:

$$
\begin{aligned}
a_{n} & =\frac{\operatorname{cov}\left(\tilde{v}, \tilde{S}_{i} \mid \hat{w}_{1}^{i}, \ldots, \hat{w}_{n-1}^{i}\right)}{\operatorname{var}\left(\tilde{S}_{i} \mid \hat{w}_{1}^{i}, \ldots, \hat{w}_{n-1}^{i}\right)} \\
& =\frac{\operatorname{cov}\left(\tilde{v}, \tilde{v}+\tilde{\epsilon}_{i} \mid p_{1}, \ldots, p_{n-1}\right)}{\operatorname{var}\left(\tilde{v}+\tilde{\epsilon}_{i} \mid p_{1}, \ldots, p_{n-1}\right)} .
\end{aligned}
$$

Then, we obtain:

$E\left[\tilde{v} \mid \tilde{S}_{i}, \tilde{w}_{1}, \ldots, \tilde{w}_{n-1}, \tilde{x}_{i 1}, \ldots, \tilde{x}_{i n-1}\right]=\frac{\Sigma_{n-1}}{\Sigma_{n-1}+\sigma_{\epsilon}^{2}}\left(\tilde{S}_{i}-\hat{p}_{n-1}^{i}\right)+\hat{p}_{n-1}^{i}=a_{n}\left(\tilde{S}_{i}-\hat{p}_{n-1}^{i}\right)+\hat{p}_{n-1}^{i}$, with $a_{n}=\frac{\Sigma_{n-1}}{\Sigma_{n-1}+\sigma_{\epsilon}^{2}}$ and $\Sigma_{n-1}=\operatorname{var}\left(\tilde{v} \mid \tilde{w}_{1}, \ldots, \tilde{w}_{n-1}\right)$ being the error variance of price at the $(n-1)^{\text {th }}$ auction.

We then compute the two terms of the profit $I$ and $I I$

$$
\begin{aligned}
I= & \tilde{x}_{i n} E\left[\tilde{v}-p_{n-1} \mid \tilde{S}_{i}, \tilde{w}_{1}, \ldots, \tilde{w}_{n-1}, \tilde{x}_{i 1}, \ldots, \tilde{x}_{i n-1}\right] \\
& -\lambda_{n} \tilde{x}_{i n} E\left[\Delta X_{n}^{*} \mid \tilde{S}_{i}, \tilde{w}_{1}, \ldots, \tilde{w}_{n-1}, \tilde{x}_{i 1}, \ldots, \tilde{x}_{i n-1}\right]-\lambda_{n} \tilde{x}_{i n}^{2}, \\
I I= & E\left[k_{1, n} \tilde{S}_{i}^{2}+k_{2, n} \tilde{S}_{i} p_{n}+k_{3, n} p_{n}^{2}+k_{4, n} p_{n}\left(\hat{p}_{n}^{i}-p_{n}\right)+k_{5, n} \tilde{S}_{i}\left(\hat{p}_{n}{ }^{i}\right.\right. \\
& \left.\left.-p_{n}\right)+k_{6, n}\left(\hat{p}_{n}^{i}-p_{n}\right)^{2}+\delta_{n} \mid \tilde{S}_{i}, \tilde{w}_{1}, \ldots, \tilde{w}_{n-1}, \tilde{x}_{i 1}, \ldots, \tilde{x}_{i n-1}\right] .
\end{aligned}
$$

Given that

$$
\begin{aligned}
& p_{n}=p_{n-1}+\lambda_{n}\left(\tilde{x}_{i n}+\Delta X_{n}^{*}+\Delta \tilde{u}_{n}\right), \\
& \hat{p}_{n}^{i}=\hat{p}_{n-1}^{i}+\lambda_{n}\left(\hat{x}_{i n}+\Delta \hat{X}_{n}^{*}+\Delta \tilde{u}_{n}\right),
\end{aligned}
$$

we obtain:

$$
\begin{aligned}
\hat{p}_{n}^{i}-p_{n} & =\hat{p}_{n-1}^{i}-p_{n-1}+\lambda_{n}\left(\hat{x}_{i n}-\tilde{x}_{i n}\right)+\lambda_{n}\left(\Delta \hat{X}_{n}^{*}-\Delta X_{n}^{*}\right) \\
& =\hat{p}_{n-1}^{i}-p_{n-1}+\lambda_{n} \hat{x}_{i n}-\lambda_{n} \tilde{x}_{i n}+(M-1) \lambda_{n} \beta_{n} \Delta t\left(\hat{p}_{n-1}^{i}-p_{n-1}\right) \\
& =\left[1+(M-1) \lambda_{n} \beta_{n} \Delta t\right]\left(\hat{p}_{n-1}^{i}-p_{n-1}\right)+\lambda_{n} \hat{x}_{i n}-\lambda_{n} \tilde{x}_{i n} .
\end{aligned}
$$


The second term $I I$ can be rewritten as

$$
\begin{aligned}
I I= & k_{1, n} \tilde{S}_{i}^{2}+k_{2, n} \tilde{S}_{i} p_{n-1}+k_{2, n} \lambda_{n} \tilde{S}_{i} E\left[\Delta X_{n}^{*} \mid \tilde{S}_{i}, \tilde{w}_{1}, \ldots, \tilde{w}_{n-1}, \tilde{x}_{i 1}, \ldots, \tilde{x}_{i n-1}\right]+k_{2, n} \lambda_{n} \tilde{S}_{i} \tilde{x}_{i n} \\
& +k_{3, n} E\left[\left(p_{n-1}+\lambda_{n} \Delta X_{n}^{*}+\lambda_{n} \tilde{x}_{i n}+\lambda_{n} \Delta \tilde{u}_{n}\right)^{2} \mid \tilde{S}_{i}, \tilde{w}_{1}, \ldots, \tilde{w}_{n-1}, \tilde{x}_{i 1}, \ldots, \tilde{x}_{i n-1}\right] \\
& +k_{4, n}\left[1+(M-1) \lambda_{n} \beta_{n} \Delta t\right] p_{n-1}\left(\hat{p}_{n-1}^{i}-p_{n-1}\right)+k_{4, n} \lambda_{n} p_{n-1}\left(\hat{x}_{i n}-\tilde{x}_{i n}\right) \\
& +k_{4, n} \lambda_{n} \tilde{x}_{i n}\left[1+(M-1) \lambda_{n} \beta_{n} \Delta t\right]\left(\hat{p}_{n-1}^{i}-p_{n-1}\right)+k_{4, n} \lambda_{n}^{2} \tilde{x}_{i n}\left(\hat{x}_{i n}-\tilde{x}_{i n}\right) \\
& +k_{4, n} \lambda_{n}\left[1+(M-1) \lambda_{n} \beta_{n} \Delta t\right]\left(\hat{p}_{n-1}^{i}-p_{n-1}\right) E\left[\Delta X_{n}^{*} \mid \tilde{S}_{i}, \tilde{w}_{1}, \ldots, \tilde{w}_{n-1}, \tilde{x}_{i 1}, \ldots, \tilde{x}_{i n-1}\right] \\
& +k_{4, n} \lambda_{n}^{2}\left(\hat{x}_{i n}-\tilde{x}_{i n}\right) E\left[\Delta X_{n}^{*} \mid \tilde{S}_{i}, \tilde{w}_{1}, \ldots, \tilde{w}_{n-1}, \tilde{x}_{i 1}, \ldots, \tilde{x}_{i n-1}\right] \\
& +k_{5, n}\left[1+(M-1) \lambda_{n} \beta_{n} \Delta t\right] \tilde{S}_{i}\left(\hat{p}_{n-1}^{i}-p_{n-1}\right) \\
& +k_{5, n} \lambda_{n} \tilde{S}_{i}\left(\hat{x}_{i n}-\tilde{x}_{i n}\right)+k_{6, n}\left(\hat{p}_{n}^{i}-p_{n}\right)^{2}+\delta_{n} .
\end{aligned}
$$

The $i t h$ informed trader chooses his market order $x_{i n}$ that maximizes his future expected profit. Thus, the first order condition (FOC) implies that:

$$
\begin{aligned}
& E\left[\tilde{v}-p_{n-1} \mid \tilde{S}_{i}, \tilde{w}_{1}, \ldots, \tilde{w}_{n-1}, \tilde{x}_{i 1}, \ldots, \tilde{x}_{i n-1}\right]-\lambda_{n} E\left[\Delta X_{n}^{*} \mid \tilde{S}_{i}, \tilde{w}_{1}, \ldots, \tilde{w}_{n-1}, \tilde{x}_{i 1}, \ldots, \tilde{x}_{i n-1}\right] \\
& E\left[k_{2, n} \lambda_{n} \tilde{S}_{i}+2 \lambda_{n} k_{3, n} p_{n}+k_{4, n}\left[\lambda_{n}\left(\hat{p}_{n}^{i}-p_{n}\right)-\lambda_{n} p_{n}\right]-\lambda_{n} k_{5, n} \tilde{S}_{i}-2 k_{6, n} \lambda_{n}\left(\hat{p}_{n}^{i}-p_{n}\right)\right. \\
& \left.\mid \tilde{S}_{i}, \tilde{w}_{1}, \ldots, \tilde{w}_{n-1}, \tilde{x}_{i 1}, \ldots, \tilde{x}_{i n-1}\right]-2 \lambda_{n} \tilde{x}_{i n}=0 .
\end{aligned}
$$

Moreover, we can directly derive the second order condition:

$$
\lambda_{n}\left[1-\lambda_{n}\left(k_{3 n}-k_{4, n}+k_{6 n}\right)\right]>0 .
$$

Given the linearity of the traders' market order, the aggregate order flow of the $j \neq i$ other informed participants is $\Delta X_{n}^{*}=\sum_{j \neq i}^{M} \alpha_{j n} \Delta t \tilde{S}_{j}+(M-1) \beta_{n} \Delta t p_{n-1}$, we have:

$$
\begin{aligned}
& E\left[\sum_{j \neq i}^{M} \alpha_{j n} \Delta t \tilde{S}_{j}+(M-1) \beta_{n} \Delta t p_{n-1} \mid \tilde{S}_{i}, \tilde{w}_{1}, \ldots, \tilde{w}_{n-1}, \tilde{x}_{i 1}, \ldots, \tilde{x}_{i n-1}\right]= \\
& \left(a_{n}\left(\tilde{S}_{i}-\hat{p}_{n-1}^{i}\right)+\hat{p}_{n-1}^{i}\right)\left(\sum_{j \neq i}^{M} \alpha_{j n} \Delta t\right)+(M-1) \beta_{n} \Delta t p_{n-1} .
\end{aligned}
$$

This leads to the following expression for the FOC: 


$$
\begin{aligned}
& a_{n}\left(\tilde{S}_{i}-\hat{p}_{n-1}^{i}\right)+\hat{p}_{n-1}^{i}-p_{n-1}-\lambda_{n}\left(a_{n}\left(\tilde{S}_{i}-\hat{p}_{n-1}^{i}\right)+\hat{p}_{n-1}^{i}\right)\left(\sum_{j \neq i}^{M} \alpha_{j n} \Delta t\right) \\
& -\lambda_{n}(M-1) \beta_{n} \Delta t p_{n-1}+k_{2 n} \lambda_{n} \tilde{S}_{i}+2 \lambda_{n} k_{3 n}\left(p_{n-1}+\lambda_{n} \tilde{x}_{i n}+\lambda_{n}\left[\left(a_{n}\left(\tilde{S}_{i}-\hat{p}_{n-1}^{i}\right)+\hat{p}_{n-1}^{i}\right)\right.\right. \\
& \left.\left.\left(\sum_{j \neq i}^{M} \alpha_{j n} \Delta t\right)+(M-1) \beta_{n} \Delta t_{n} p_{n-1}\right]\right)+k_{4 n} \lambda_{n}\left[1+(M-1) \lambda_{n} \beta_{n} \Delta t\right]\left(\hat{p}_{n-1}^{i}-p_{n-1}\right) \\
& +k_{4 n} \lambda_{n}^{2}\left(\hat{x}_{i n}-\tilde{x}_{i n}\right)-k_{4 n} \lambda_{n}\left(p_{n-1}+\lambda_{n} \tilde{x}_{i n}+\lambda_{n}\left[\left(a_{n}\left(\tilde{S}_{i}-\hat{p}_{n-1}^{i}\right)+\hat{p}_{n-1}^{i}\right)\right.\right. \\
& \left.\left.\left(\sum_{j \neq i}^{M} \alpha_{j n} \Delta t\right)+(M-1) \beta_{n} \Delta t_{n} p_{n-1}\right]\right)-k_{5 n} \lambda_{n} \tilde{S}_{i} \\
& -2 k_{6 n} \lambda_{n}\left[1+(M-1) \lambda_{n} \beta_{n} \Delta t\right]\left(\hat{p}_{n-1}^{i}-p_{n-1}\right)-2 k_{6 n} \lambda_{n}^{2}\left(\hat{x}_{i n}-\tilde{x}_{i n}\right)-2 \lambda_{n} \tilde{x}_{i n}=0 .
\end{aligned}
$$

The FOC can be rewritten as:

$$
\begin{gathered}
\tilde{S}_{i}\left[a_{n}-2 \lambda_{n}\left(1-\lambda_{n}\left(k_{3 n}-\frac{1}{2} k_{4 n}\right)\right) \alpha_{i n} \Delta t+\lambda_{n}\left(k_{2 n}-k_{5 n}\right)+\lambda_{n} a_{n}\left(2 \lambda_{n}\left(k_{3 n}-\frac{1}{2} k_{4 n}\right)-1\right)\right. \\
\left.\left(\sum_{j \neq i}^{M} \alpha_{j n} \Delta t\right)\right]+p_{n-1}\left[-a_{n}+2 \lambda_{n}\left(k_{3 n}-\frac{1}{2} k_{4 n}\right)-\left(M+1-2 M \lambda_{n}\left(k_{3 n}-\frac{1}{2} k_{4 n}\right)\right) \lambda_{n} \beta_{n} \Delta t\right. \\
\left.-\lambda_{n}\left(1-a_{n}\right)\left(1-2 \lambda_{n}\left(k_{3 n}-\frac{1}{2} k_{4 n}\right)\right)\left(\sum_{j \neq i}^{M} \alpha_{j n} \Delta t\right)\right]+\left(\hat{p}_{n-1}^{i}-p_{n-1}\right)\left[1-a_{n}\right. \\
+\left(1-a_{n}\right) \lambda_{n}\left(2 \lambda_{n}\left(k_{3 n}-\frac{1}{2} k_{4 n}\right)-1\right)\left(\sum_{j \neq i}^{M} \alpha_{j n} \Delta t\right)+2 \lambda_{n}\left(\beta_{n}+\zeta_{n}\right) \Delta t\left(\lambda_{n}\left(k_{3 n}-\frac{1}{2} k_{4 n}\right)-1\right) \\
\left.-2 \lambda_{n}^{2} \beta_{n} \Delta t(M-1)\left(k_{6 n}-\frac{1}{2} k_{4 n}\right)+2 \lambda_{n}^{2} \zeta_{n} \Delta t\left(k_{6 n}-\frac{1}{2} k_{4 n}\right)-2 \lambda_{n}\left(k_{6 n}-\frac{1}{2} k_{4 n}\right)\right]=0 .
\end{gathered}
$$

By identification, the coefficients multiplied by $\tilde{S}_{i}, p_{n-1}$ and $\hat{p}_{n-1}^{i}-p_{n-1}$ must be equal to zero. This leads to:

$a_{n}-2 \lambda_{n}\left[1-\lambda_{n}\left(k_{3 n}-\frac{1}{2} k_{4 n}\right)\right] \alpha_{i n} \Delta t+\lambda_{n}\left(k_{2 n}-k_{5 n}\right)+\lambda_{n} a_{n}\left[2 \lambda_{n}\left(k_{3 n}-\frac{1}{2} k_{4 n}\right)-1\right]\left(\sum_{j \neq i}^{M} \alpha_{j n} \Delta t_{n}\right)=0$.

After some further simplifications we obtain

$$
\begin{gathered}
-a_{n}+2 \lambda_{n}\left(k_{3 n}-\frac{1}{2} k_{4 n}\right)-\left[M+1-2 M \lambda_{n}\left(k_{3 n}-\frac{1}{2} k_{4 n}\right)\right] \lambda_{n} \beta_{n} \Delta t \\
-\lambda_{n}\left(1-a_{n}\right)\left[1-2 \lambda_{n}\left(k_{3 n}-\frac{1}{2} k_{4 n}\right)\right]\left(\sum_{j \neq i}^{M} \alpha_{j n} \Delta t\right)=0,
\end{gathered}
$$

and

$$
\begin{gathered}
1-a_{n}+\left(1-a_{n}\right) \lambda_{n}\left[2 \lambda_{n}\left(k_{3 n}-\frac{1}{2} k_{4 n}\right)-1\right]\left(\sum_{j \neq i}^{M} \alpha_{j n} \Delta t\right)+2 \lambda_{n}\left(\beta_{n}+\zeta_{n}\right) \Delta t\left[\lambda_{n}\left(k_{3 n}-\frac{1}{2} k_{4 n}\right)-1\right] \\
-2 \lambda_{n}^{2} \beta_{n} \Delta t(M-1)\left(k_{6 n}-\frac{1}{2} k_{4 n}\right)+2 \lambda_{n}^{2} \zeta_{n} \Delta t\left(k_{6 n}-\frac{1}{2} k_{4 n}\right)-2 \lambda_{n}\left(k_{6 n}-\frac{1}{2} k_{4 n}\right)=0 .
\end{gathered}
$$


We then obtain the following relationships for $\alpha_{i n}, \beta_{n}$ and $\zeta_{n}$

$$
\begin{gathered}
\alpha_{i n} \Delta t=\frac{a_{n}+\lambda_{n}\left(k_{2 n}-k_{5 n}\right)}{2 \lambda_{n}\left[1-\lambda_{n}\left(k_{3 n}-\frac{1}{2} k_{4 n}\right)\right]}+a_{n} \frac{2 \lambda_{n}\left(k_{3 n}-\frac{1}{2} k_{4 n}\right)-1}{2\left[1-\lambda_{n}\left(k_{3 n}-\frac{1}{2} k_{4 n}\right)\right]}\left(\sum_{j \neq i}^{M} \alpha_{j n} \Delta t\right), \\
\beta_{n} \Delta t=\frac{2\left(k_{3 n}-\frac{1}{2} k_{4 n}\right) \lambda_{n}-a_{n}}{(M+1) \lambda_{n}-2 M\left(k_{3 n}-\frac{1}{2} k_{4 n}\right) \lambda_{n}^{2}}-\frac{\lambda_{n}\left(1-a_{n}\right)\left[1-2 \lambda_{n}\left(k_{3 n}-\frac{1}{2} k_{4 n}\right)\right]}{(M+1) \lambda_{n}-2 M\left(k_{3 n}-\frac{1}{2} k_{4 n}\right) \lambda_{n}^{2}} \sum_{j \neq i}^{M} \alpha_{j n} \Delta t, \\
\zeta_{n} \Delta t=\frac{a_{n}-1+2 \lambda_{n}\left(k_{6 n}-\frac{1}{2} k_{4 n}\right)-\left(1-a_{n}\right) \lambda_{n}\left[2 \lambda_{n}\left(k_{3 n}-\frac{1}{2} k_{4 n}\right)-1\right]\left(\sum_{j \neq i}^{M} \alpha_{j n} \Delta t\right)}{2 \lambda_{n}\left[\lambda_{n}\left(k_{3 n}-\frac{1}{2} k_{4 n}\right)-1\right]+2 \lambda_{n}^{2}\left(k_{6 n}-\frac{1}{2} k_{4 n}\right)} \\
-\frac{2 \lambda_{n}\left[\lambda_{n}\left(k_{3 n}-\frac{1}{2} k_{4 n}\right)-1\right]-2 \lambda_{n}^{2}(M-1)\left(k_{6 n}-\frac{1}{2} k_{4 n}\right)}{2 \lambda_{n}\left[\lambda_{n}\left(k_{3 n}-\frac{1}{2} k_{4 n}\right)-1\right]+2 \lambda_{n}^{2}\left(k_{6 n}-\frac{1}{2} k_{4 n}\right)} \beta_{n} \Delta t .
\end{gathered}
$$

The first relationship needs to be solved for the $\alpha_{i n}$ parameters. Let us define the following parameters

$$
a^{\prime}=\frac{a_{n}+\lambda_{n}\left(k_{2 n}-k_{5 n}\right)}{2 \lambda_{n}\left[1-\lambda_{n}\left(k_{3 n}-\frac{1}{2} k_{4 n}\right)\right]}, \text { and } b^{\prime}=-\frac{a_{n}}{2} \frac{1-2 \lambda_{n}\left(k_{3 n}-\frac{1}{2} k_{4 n}\right)}{1-\lambda_{n}\left(k_{3 n}-\frac{1}{2} k_{4 n}\right)} .
$$

Given $a^{\prime}$ and $b^{\prime}$, the relationship (6.4) between the parameters $\alpha_{i}$ can be rewritten as, for $i \neq j$

$$
\alpha_{i}=a^{\prime}+b^{\prime}\left(\sum_{j \neq i}^{n} \alpha_{j}\right) .
$$

\section{Step 3: The demand of the insiders.}

The Lemma below gives the expression of the $\alpha_{i}$ parameters solving that relationship.

Lemma Let $a^{\prime}$ and $b^{\prime}$ be two real numbers such as for $i \neq j$ the relationship (6.7) is verified then, if $b^{\prime} \neq-1$ for $i=1, \ldots, M$ :

$$
\alpha_{i}=\frac{a^{\prime}}{1-b^{\prime}(M-1)}
$$

Proof: We have the following $M$ equalities

$$
\begin{aligned}
\alpha_{1}= & a^{\prime}+b^{\prime}\left(\alpha_{2}+\ldots+\alpha_{M}\right), \\
\alpha_{2}= & a^{\prime}+b^{\prime}\left(\alpha_{1}+\alpha_{3}+\ldots+\alpha_{M}\right), \\
& \vdots \\
\alpha_{M}= & a^{\prime}+b^{\prime}\left(\alpha_{1}+\alpha_{2}+\ldots+\alpha_{M-1}\right) .
\end{aligned}
$$


Let $t$ be a real number such that $t=\sum_{i=1}^{M} \alpha_{i}$, by adding the $M$ previous equalities, we get:

$$
\begin{gathered}
t=M a^{\prime}+b^{\prime}(M-1) t, \\
t=\frac{M a^{\prime}}{1-b^{\prime}(M-1)} .
\end{gathered}
$$

On the other hand, by considering the difference of the first two equalities we have:

$$
\alpha_{2}-\alpha_{1}=b^{\prime}\left(\alpha_{1}-\alpha_{2}\right)
$$

Hence, we obtain:

$$
\alpha_{2}\left(1+b^{\prime}\right)=\alpha_{1}\left(1+b^{\prime}\right)
$$

Then, if $b^{\prime} \neq-1$, all the real numbers $\alpha_{i}$ are identical.

Therefore, we can conclude for $i=1, \ldots, M$ :

$$
\alpha_{i}=\frac{a^{\prime}}{1-b^{\prime}(M-1)}
$$

It can be verified that the case where $b^{\prime}=-1$, cannot happen due to the second order condition.

This ends the proof of the lemma.

By applying the lemma, we find the following expression of $\alpha_{i n} \Delta t$ which is independent of $i$ :

$$
\alpha_{i n} \Delta t=\alpha_{n} \Delta t=\frac{a_{n}+\lambda_{n}\left(k_{2 n}-k_{5 n}\right)}{\lambda_{n}\left[2+(M-1) a_{n}-2 \lambda_{n}\left(k_{3 n}-\frac{1}{2} k_{4 n}\right)\left(1+a_{n}(M-1)\right)\right]} .
$$

The expression of $\beta_{n}$ is given by:

$$
\beta_{n} \Delta t=\frac{2\left(k_{3 n}-\frac{1}{2} k_{4 n}\right) \lambda_{n}-a_{n}}{(M+1) \lambda_{n}-2 M\left(k_{3 n}-\frac{1}{2} k_{4 n}\right) \lambda_{n}^{2}}-\frac{M-1}{M} \frac{\left(1-a_{n}\right)\left(1-2 \lambda_{n}\left(k_{3 n}-\frac{1}{2} k_{4 n}\right)\right)}{(M+1) \lambda_{n}-2 M\left(k_{3 n}-\frac{1}{2} k_{4 n}\right) \lambda_{n}^{2}} \psi_{n},
$$

with $\psi_{n}=M \lambda_{n} \alpha_{n} \Delta t$.

On the other hand, one obtains the relationship between the error variance of prices at the $n t h$ auction $\left(\Sigma_{n}\right)$ and the error variance of prices at the $(n-1)$ th auction. Indeed:

$$
\Sigma_{n}=\operatorname{var}\left[\tilde{v} \mid \tilde{w}_{1}, \ldots, \tilde{w}_{n}\right]=\operatorname{var}\left[\tilde{v} \mid \tilde{w}_{1}, \ldots, \tilde{w}_{n-1}\right]-\frac{\operatorname{cov}\left(\tilde{v}, \tilde{w}_{n}\right)^{2}}{\operatorname{var}\left(\tilde{w}_{n}\right)}
$$


Hence, one obtains:

$$
\Sigma_{n}=\Sigma_{n-1}-\lambda_{n} \operatorname{cov}\left(\tilde{v}, \tilde{w}_{n}\right)=\left(1-M \lambda_{n} \alpha_{n} \Delta t\right) \Sigma_{n-1}=\left(1-\psi_{n}\right) \Sigma_{n-1} .
$$

Since $\Sigma_{n}$ is positive, we have the following condition:

$$
\psi_{n}<1 \text {. }
$$

Then, $a_{n}$ can be written as:

$$
a_{n}=\frac{\Sigma_{n-1}}{\Sigma_{n-1}+\sigma_{\epsilon}^{2}}=\frac{\Sigma_{n}}{\Sigma_{n}+\left(1-\psi_{n}\right) \sigma_{\epsilon}^{2}} .
$$

The error variance of the price at the $n t h$ auction, $\Sigma_{n}$, is equal to:

$$
\begin{gathered}
\Sigma_{n}=\operatorname{var}\left[\tilde{v} \mid \tilde{w}_{1}, \ldots, \tilde{w}_{n}\right]=\Sigma_{n-1}-\frac{\left(\alpha_{n} \Delta t\right)^{2} \Sigma_{n-1}^{2} M^{2}}{\left(\alpha_{n} \Delta t\right)^{2} \Sigma_{n-1} M^{2}+M\left(\alpha_{n} \Delta t\right)^{2} \sigma_{\epsilon}^{2}+\sigma_{u}^{2} \Delta t}, \\
\Sigma_{n}=\frac{\Sigma_{n-1}\left(\sigma_{u}^{2} \Delta t+\left(\alpha_{n} \Delta t\right)^{2} M \sigma_{\epsilon}^{2}\right)}{\left(\alpha_{n} \Delta t\right)^{2} M^{2} \Sigma_{n-1}+\sigma_{u}^{2} \Delta t+\left(\alpha_{n} \Delta t\right)^{2} M \sigma_{\epsilon}^{2}} .
\end{gathered}
$$

The market efficiency condition implies that $\lambda_{n}$ is the regression coefficient of $\tilde{v}$ on $\tilde{w}_{n}$, conditional on $\tilde{w}_{1}, \ldots, \tilde{w}_{n-1}$, then:

$$
\lambda_{n}=\frac{\alpha_{n} \Delta t M \Sigma_{n-1}}{\left(\alpha_{n} \Delta t_{n}\right)^{2} M^{2} \Sigma_{n-1}+\sigma_{u}^{2} \Delta t+\left(\alpha_{n} \Delta t_{n}\right)^{2} M \sigma_{\epsilon}^{2}} .
$$

This leads to the following expression

$$
\frac{\lambda_{n}}{\Sigma_{n}}=\frac{M \alpha_{n} \Delta t}{M\left(\alpha_{n} \Delta t\right)^{2} \sigma_{\epsilon}^{2}+\sigma_{u}^{2} \Delta t} .
$$

Since $\alpha_{n} \Delta t=\frac{\psi_{n}}{M \lambda_{n}}$, one obtains:

$$
\lambda_{n}^{2}=\frac{\psi_{n} \Sigma_{n}-\frac{\psi_{n}^{2} \sigma_{\epsilon}^{2}}{M}}{\sigma_{u}^{2} \Delta t} .
$$

That yields the following condition:

$$
\Sigma_{n}>\psi_{n} \frac{\sigma_{\epsilon}^{2}}{M}
$$

Since $\Sigma_{n} \geq \Sigma_{N}$ and $\psi_{n}<1$, the following condition is sufficient for equilibrium

$$
\Sigma_{N}>\frac{\sigma_{\epsilon}^{2}}{M}
$$




\section{Step 4: The backward induction program of the insiders.}

Each trader $i$, for $i=1, \ldots, M$, solves his maximization problem:

$$
\begin{aligned}
E\left[\pi_{i n} \mid p_{1}, \ldots, p_{n-1}, \tilde{S}_{i}\right]= & k_{1, n-1} \tilde{S}_{i}^{2}+k_{2, n-1} \tilde{S}_{i} p_{n-1}+k_{3, n-1} p_{n-1}^{2}+k_{4, n-1} p_{n-1}\left(\hat{p}_{n-1}^{i}-p_{n-1}\right) \\
& +k_{5, n-1} \tilde{S}_{i}\left(\hat{p}_{n-1}^{i}-p_{n-1}\right)+k_{6, n-1}\left(\hat{p}_{n-1}^{i}-p_{n-1}\right)^{2}+\delta_{n-1} .
\end{aligned}
$$

Since each trader uses a backward induction process, we have to find a recurrence relation between the different parameters:

$$
\left(\begin{array}{l}
k_{1, n-1} \\
k_{2, n-1} \\
k_{3, n-1} \\
k_{4, n-1} \\
k_{5, n-1} \\
k_{6, n-1}
\end{array}\right)=\left(\begin{array}{lllllll}
a_{11, n} & a_{12, n} & a_{13, n} & a_{14, n} & a_{15, n} & a_{16, n} \\
a_{21, n} & a_{22, n} & a_{23, n} & a_{24, n} & a_{25, n} & a_{26, n} \\
a_{31, n} & a_{32, n} & a_{33, n} & a_{34, n} & a_{35, n} & a_{36, n} \\
a_{41, n} & a_{42, n} & a_{43, n} & a_{44, n} & a_{45, n} & a_{46, n} \\
a_{51, n} & a_{52, n} & a_{53, n} & a_{54, n} & a_{55, n} & a_{56, n} \\
a_{61, n} & a_{62, n} & a_{63, n} & a_{64, n} & a_{65, n} & a_{66, n}
\end{array}\right)\left(\begin{array}{c}
k_{1, n} \\
k_{2, n} \\
k_{3, n} \\
k_{4, n} \\
k_{5, n} \\
k_{6, n}
\end{array}\right)+\left(\begin{array}{c}
c_{1, n} \\
c_{2, n} \\
c_{3, n} \\
c_{4, n} \\
c_{5, n} \\
c_{6, n}
\end{array}\right) .
$$

In order to find the expression of the profit of the $i t h$ informed trader, we calculate $E\left[\Delta X_{n}^{*} \mid \tilde{S}_{i}, \tilde{w}_{1}, \ldots, \tilde{w}_{n-1}, \tilde{x}_{i 1}, \ldots, \tilde{x}_{i n-1}\right]$ and $E\left[\left(\Delta X_{n}^{*}\right)^{2} \mid \tilde{S}_{i}, \tilde{w}_{1}, \ldots, \tilde{w}_{n-1}, \tilde{x}_{i 1}, \ldots, \tilde{x}_{i n-1}\right]$. They are given by

$$
\begin{gathered}
E\left[\Delta X_{n}^{*} \mid \tilde{S}_{i}, \tilde{w}_{1}, \ldots, \tilde{w}_{n-1}, \tilde{x}_{i 1}, \ldots, \tilde{x}_{i n-1}\right]=(M-1) \alpha_{n} \Delta t a_{n} \tilde{S}_{i} \\
+(M-1)\left[\beta_{n} \Delta t+\alpha_{n} \Delta t_{n}\left(1-a_{n}\right)\right] p_{n-1}+(M-1)\left(1-a_{n}\right) \alpha_{n} \Delta t\left(\hat{p}_{n-1}^{i}-p_{n-1}\right),
\end{gathered}
$$

and

$$
\begin{gathered}
E\left[\left(\Delta X_{n}^{*}\right)^{2} \mid \tilde{S}_{i}, \tilde{w}_{1}, \ldots, \tilde{w}_{n-1}, \tilde{x}_{i 1}, \ldots, \tilde{x}_{i n-1}\right]=E\left[(M-1)^{2}\left(\beta_{n} \Delta t_{n}\right)^{2} p_{n-1}^{2}\right. \\
\left.+\left(\alpha_{n} \Delta t\right)^{2}\left(\sum_{j \neq i}^{M} \tilde{S}_{j}\right)^{2}+2 \alpha_{n} \Delta t \beta_{n} \Delta t(M-1) p_{n-1}\left(\sum_{j \neq i}^{M} \tilde{S}_{j}\right) \mid \tilde{S}_{i}, \tilde{w}_{1}, \ldots, \tilde{w}_{n-1}, \tilde{x}_{i 1}, \ldots, \tilde{x}_{i n-1}\right] .
\end{gathered}
$$

It remains to calculate $E\left[\left(\sum_{j \neq i}^{M} \tilde{S}_{j}\right)^{2} \mid \tilde{S}_{i}, \tilde{w}_{1}, \ldots, \tilde{w}_{n-1}, \tilde{x}_{i 1}, \ldots, \tilde{x}_{i n-1}\right]$. That expression can 
be written as:

$$
\begin{array}{r}
E\left[\left(\sum_{j \neq i}^{M} \tilde{S}_{j}\right)^{2} \mid \tilde{S}_{i}, \tilde{w}_{1}, \ldots, \tilde{w}_{n-1}, \tilde{x}_{i 1}, \ldots, \tilde{x}_{i n-1}\right]=E\left[\sum_{j \neq i} \tilde{S}_{j}^{2}+2 \sum_{j<k} \tilde{S}_{j} \tilde{S}_{k} \mid \tilde{S}_{i}, \tilde{w}_{1}, \ldots, \tilde{w}_{n-1}\right. \\
\left., \tilde{x}_{i 1}, \ldots, \tilde{x}_{i n-1}\right] .
\end{array}
$$

We then have

$$
\begin{aligned}
E\left[\tilde{S}_{j} \tilde{S}_{k} \mid \tilde{S}_{i}, \tilde{w}_{1}, \ldots, \tilde{w}_{n-1}, \tilde{x}_{i 1}, \ldots, \tilde{x}_{i n-1}\right] & =E\left[\left(\tilde{v}+\tilde{\epsilon}_{j}\right)\left(\tilde{v}+\tilde{\epsilon}_{k}\right) \mid \tilde{S}_{i}, \tilde{w}_{1}, \ldots, \tilde{w}_{n-1}, \tilde{x}_{i 1}, \ldots, \tilde{x}_{i n-1}\right] \\
& =E\left[\tilde{v}^{2} \mid \tilde{S}_{i}, \tilde{w}_{1}, \ldots, \tilde{w}_{n-1}, \tilde{x}_{i 1}, \ldots, \tilde{x}_{i n-1}\right] \\
& =E\left[\tilde{v}^{2} \mid \tilde{S}_{i}, \hat{w}_{1}^{i}, \ldots, \hat{w}_{n-1}^{i}, \tilde{x}_{i 1}, \ldots, \tilde{x}_{i n-1}\right] \\
& =E\left[\tilde{v}^{2} \mid \tilde{S}_{i}, \hat{w}_{1}^{i}, \ldots, \hat{w}_{n-1}^{i}\right],
\end{aligned}
$$

and

$$
\begin{aligned}
\operatorname{Var}\left(\tilde{v} \mid \tilde{S}_{i}, \hat{w}_{1}^{i}, \ldots, \hat{w}_{n-1}^{i}\right) & =\operatorname{Var}\left(\tilde{v} \mid \hat{w}_{1}^{i}, \ldots, \hat{w}_{n-1}^{i}\right)-\frac{\left[\operatorname{Cov}\left(\tilde{v}, \tilde{S}_{i} \mid \hat{w}_{1}^{i}, \ldots, \hat{w}_{n-1}^{i}\right)\right]^{2}}{\operatorname{Var}\left(\tilde{S}_{i} \mid \hat{w}_{1}^{i}, \ldots, \hat{w}_{n-1}^{i}\right)} \\
& =\Sigma_{n-1}-\frac{\Sigma_{n-1}^{2}}{\Sigma_{n-1}+\sigma_{\epsilon}^{2}}=a_{n} \sigma_{\epsilon}^{2} .
\end{aligned}
$$

Given the above, we get:

$$
\begin{aligned}
E\left[\tilde{S}_{j} \tilde{S}_{k} \mid \tilde{S}_{i}, \tilde{w}_{1}, \ldots, \tilde{w}_{n-1}, \tilde{x}_{i 1}, \ldots, \tilde{x}_{i n-1}\right] & \left.=E\left[\tilde{v}_{\mid} \tilde{S}_{i}, \tilde{w}_{1}, \ldots, \tilde{w}_{n-1}, \tilde{x}_{i 1}, \ldots, \tilde{x}_{i n-1}\right]^{2}+a_{(6,60 \hat{\epsilon}}\right) \\
& =\left[a_{n}\left(\tilde{S}_{i}-\hat{p}_{n-1}^{i}\right)+\hat{p}_{n-1}^{i}\right]^{2}+a_{n} \sigma_{\epsilon}^{2} .
\end{aligned}
$$

We then obtain that

$$
\begin{aligned}
& E\left[\tilde{S}_{j} \tilde{S}_{k} \mid \tilde{S}_{i}, \tilde{w}_{1}, \ldots, \tilde{w}_{n-1}, \tilde{x}_{i 1}, \ldots, \tilde{x}_{i n-1}\right]=a_{n}^{2} \tilde{S}_{i}^{2}+\left(1-a_{n}\right)^{2} p_{n-1}^{2}+\left(1-a_{n}\right)^{2}\left(\hat{p}_{n-1}^{i}-p_{n-1}\right)^{2} \\
+ & 2\left(1-a_{n}\right)^{2} p_{n-1}\left(\hat{p}_{n-1}^{i}-p_{n-1}\right)+2 a_{n}\left(1-a_{n}\right) \tilde{S}_{i}\left(\hat{p}_{n-1}^{i}-p_{n-1}\right)+2 a_{n}\left(1-a_{n}\right) \tilde{S}_{i} p_{n-1}+a_{n} \sigma_{\epsilon}^{2},
\end{aligned}
$$

and

$$
\begin{gathered}
E\left[\tilde{S}_{j}^{2} \mid \tilde{S}_{i}, \tilde{w}_{1}, \ldots, \tilde{w}_{n-1}, \tilde{x}_{i 1}, \ldots, \tilde{x}_{i n-1}\right]=a_{n}^{2} \tilde{S}_{i}^{2}+\left(1-a_{n}\right)^{2} p_{n-1}^{2}+\left(1-a_{n}\right)^{2}\left(\hat{p}_{n-1}^{i}-p_{n-1}\right)^{2} \\
+2\left(1-a_{n}\right)^{2} p_{n-1}\left(\hat{p}_{n-1}^{i}-p_{n-1}\right)+2 a_{n}\left(1-a_{n}\right) \tilde{S}_{i}\left(\hat{p}_{n-1}^{i}-p_{n-1}\right)+2 a_{n}\left(1-a_{n}\right) \tilde{S}_{i} p_{n-1}+\left(a_{n}+1\right) \sigma_{\epsilon}^{2} .
\end{gathered}
$$


Using the two previous expressions to compute (6.9), leads to:

$$
\begin{gathered}
E\left[\left(\sum_{j \neq i}^{M} \tilde{S}_{j}\right)^{2} \mid \tilde{S}_{i}, \tilde{w}_{1}, \ldots, \tilde{w}_{n-1}, \tilde{x}_{i 1}, \ldots, \tilde{x}_{i n-1}\right]=a_{n}^{2}(M-1)^{2} \tilde{S}_{i}^{2}+\left(1-a_{n}\right)^{2}(M-1)^{2} p_{n-1}^{2} \\
\quad+\left(1-a_{n}\right)^{2}(M-1)^{2}\left(\hat{p}_{n-1}^{i}-p_{n-1}\right)^{2}+2\left(1-a_{n}\right)^{2}(M-1)^{2} p_{n-1}\left(\hat{p}_{n-1}^{i}-p_{n-1}\right) \\
+2 a_{n}\left(1-a_{n}\right)(M-1)^{2} \tilde{S}_{i}\left(\hat{p}_{n-1}^{i}-p_{n-1}\right)+2 a_{n}\left(1-a_{n}\right)(M-1)^{2} \tilde{S}_{i} p_{n-1}+(M-1)\left[1+(M-1) a_{n}\right] \sigma_{\epsilon}^{2} .
\end{gathered}
$$

This is then used to calculate expression (6.8) and leads to

$$
\begin{aligned}
E\left[\left(\Delta X_{n}^{*}\right)^{2} \mid \tilde{S}_{i}, \tilde{w}_{1}, \ldots, \tilde{w}_{n-1}, \tilde{x}_{i 1}, \ldots, \tilde{x}_{i n-1}\right]= & \left(\alpha_{n} \Delta t_{n}\right)^{2} a_{n}^{2}(M-1)^{2} \tilde{S}_{i}^{2} \\
& +(M-1)^{2}\left(\alpha_{n} \Delta t\left(1-a_{n}\right)+\beta_{n} \Delta t_{n}\right)^{2} p_{n-1}^{2} \\
& +\left(\alpha_{n} \Delta t\right)^{2}\left(1-a_{n}\right)^{2}(M-1)^{2}\left(\hat{p}_{n-1}^{i}-p_{n-1}\right)^{2} \\
& +2 \alpha_{n} \Delta t\left(1-a_{n}\right)(M-1)^{2}\left[\alpha_{n} \Delta t_{n}\left(1-a_{n}\right)\right. \\
& \left.+\beta_{n} \Delta t\right] p_{n-1}\left(\hat{p}_{n-1}^{i}-p_{n-1}\right) \\
& +2 a_{n}\left(1-a_{n}\right)\left(\alpha_{n} \Delta t\right)^{2}(M-1)^{2} \tilde{S}_{i}\left(\hat{p}_{n-1}^{i}-p_{n-1}\right) \\
& +2 a_{n} \alpha_{n} \Delta t(M-1)^{2}\left[\left(1-a_{n}\right) \alpha_{n} \Delta t\right. \\
& \left.+\beta_{n} \Delta t\right] \tilde{S}_{i} p_{n-1} \\
& +\left(\alpha_{n} \Delta t\right)^{2}(M-1)\left[1+(M-1) a_{n}\right] \sigma_{\epsilon}^{2} .
\end{aligned}
$$

We calculate the profit of the $i t h$ informed trader by substituting $\tilde{x}_{i n}=\alpha_{n} \Delta t \tilde{S}_{i}+\beta_{n} \Delta t p_{n-1}+$ $\zeta_{n} \Delta t\left(\hat{p}_{n-1}^{i}-p_{n-1}\right)$ in the expression of the profit, and by identification we obtain the different coefficients:

$$
\left.\delta_{n-1}=\delta_{n}+\lambda_{n}^{2} k_{3 n}\left[\sigma_{u}^{2} \Delta t_{n}+(M-1)\left(\alpha_{n} \Delta t\right)^{2}\left(1+(M-1) a_{n}\right) \sigma_{\epsilon}^{2}\right]\right]
$$




$$
\begin{aligned}
& \left\{\begin{array}{l}
a_{11, n}=1, \\
a_{12, n}=\lambda_{n}\left(\alpha_{n} \Delta t\right)\left[1+(M-1) a_{n}\right], \\
a_{13, n}=\lambda_{n}^{2}\left(\alpha_{n} \Delta t\right)^{2}\left[1+(M-1) a_{n}\right]^{2},
\end{array}\right. \\
& a_{22, n}=1+\lambda_{n} \beta_{n} \Delta t+\lambda_{n}(M-1)\left[\beta_{n} \Delta t+\alpha_{n} \Delta t\left(1-a_{n}\right)\right], \\
& a_{23, n}=2 \lambda_{n} \alpha_{n} \Delta t\left[1+a_{n}(M-1)\right]\left[1+M \lambda_{n} \beta_{n} \Delta t+(M-1)\left(1-a_{n}\right) \lambda_{n} \alpha_{n} \Delta t_{n}\right], \\
& a_{33, n}=\left[1+\lambda_{n}\left((M-1) \alpha_{n} \Delta t\left(1-a_{n}\right)+M \beta_{n} \Delta t\right)\right]^{2}, \\
& a_{43, n}=2 \lambda_{n}^{2} \alpha_{n} \Delta t\left(1-a_{n}\right)(M-1)^{2}\left[\alpha_{n} \Delta t\left(1-a_{n}\right)+\beta_{n} \Delta t\right]+2 \lambda_{n}^{2} \beta_{n} \Delta t \zeta_{n} \Delta t \\
& +2 \lambda_{n}\left(1-a_{n}\right)(M-1) \alpha_{n} \Delta t+2 \lambda_{n} \zeta_{n} \Delta t+2 \lambda_{n}^{2}\left[\left(1-a_{n}\right)(M-1) \alpha_{n} \Delta t_{n} \beta_{n} \Delta t\right. \\
& \left.+(M-1) \zeta_{n} \Delta t\left(\alpha_{n} \Delta t\left(1-a_{n}\right)+\beta_{n} \Delta t\right)\right] \\
& a_{44, n}=1+(M-1) \lambda_{n} \beta_{n} \Delta t+\lambda_{n}\left(\beta_{n} \Delta t-\zeta_{n} \Delta t\right)+\lambda_{n}\left[1+(M-1) \lambda_{n} \beta_{n} \Delta t\right] \beta_{n} \Delta t \\
& +\lambda_{n}^{2} \beta_{n} \Delta t\left(\beta_{n} \Delta t-\zeta_{n} \Delta t\right)+\lambda_{n}\left[1+(M-1) \lambda_{n} \beta_{n} \Delta t_{n}\right](M-1)\left[\alpha_{n} \Delta t\left(1-a_{n}\right)+\beta_{n} \Delta t\right] \\
& +\lambda_{n}^{2}\left(\beta_{n} \Delta t-\zeta_{n} \Delta t\right)(M-1)\left[\alpha_{n} \Delta t\left(1-a_{n}\right)+\beta_{n} \Delta t\right] \\
& a_{52, n}=\lambda_{n}\left(1-a_{n}\right)(M-1) \alpha_{n} \Delta t+\lambda_{n} \zeta_{n} \Delta t, \\
& a_{53, n}=2 \lambda_{n}^{2} a_{n}\left(1-a_{n}\right)\left(\alpha_{n} \Delta t_{n}\right)^{2}(M-1)^{2}+2 \lambda_{n}^{2} \alpha_{n} \Delta t \zeta_{n} \Delta t_{n} \\
& +2 \lambda_{n}^{2}\left[\left(1-a_{n}\right)(M-1)\left(\alpha_{n} \Delta t_{n}\right)^{2}+a_{n}(M-1) \alpha_{n} \Delta t \zeta_{n} \Delta t\right], \\
& a_{54, n}=\lambda_{n}\left[1+(M-1) \lambda_{n} \beta_{n} \Delta t\right] \alpha_{n} \Delta t+\lambda_{n}^{2}\left(\beta_{n} \Delta t-\zeta_{n} \Delta t_{n}\right) \alpha_{n} \Delta t \\
& +\lambda_{n}\left[1+(M-1) \lambda_{n} \beta_{n} \Delta t\right] a_{n}(M-1) \alpha_{n} \Delta t+\lambda_{n}^{2}\left(\beta_{n} \Delta t-\zeta_{n} \Delta t_{n}\right) a_{n}(M-1) \alpha_{n} \Delta t, \\
& a_{55, n}=1+(M-1) \lambda_{n} \beta_{n} \Delta t+\lambda_{n}\left(\beta_{n} \Delta t-\zeta_{n} \Delta t\right), \\
& a_{63, n}=\lambda_{n}^{2}\left[\zeta_{n} \Delta t+\left(\alpha_{n} \Delta t_{n}\right)\left(1-a_{n}\right)(M-1)\right]^{2}, \\
& a_{64, n}=\lambda_{n}\left[1+(M-1) \lambda_{n} \beta_{n} \Delta t\right] \zeta_{n} \Delta t+\lambda_{n}^{2} \zeta_{n} \Delta t\left(\beta_{n} \Delta t_{n}-\zeta_{n} \Delta t\right) \\
& +\lambda_{n}\left[1+(M-1) \lambda_{n} \beta_{n} \Delta t\right]\left(1-a_{n}\right)(M-1) \alpha_{n} \Delta t \\
& +\lambda_{n}^{2}\left(\beta_{n} \Delta t-\zeta_{n} \Delta t_{n}\right)\left(1-a_{n}\right)(M-1) \alpha_{n} \Delta t, \\
& a_{66, n}=\left[\left(1+(M-1) \lambda_{n} \beta_{n} \Delta t\right)+\lambda_{n}\left(\beta_{n} \Delta t-\zeta_{n} \Delta t\right)\right]^{2},
\end{aligned}
$$

and 


$$
\left\{\begin{array}{l}
c_{1 n}=\alpha_{n} \Delta t\left(a_{n}-(M-1) a_{n} \lambda_{n} \alpha_{n} \Delta t_{n}-\lambda_{n} \alpha_{n} \Delta t\right), \\
c_{2 n}=a_{n}\left(\beta_{n} \Delta t-\alpha_{n} \Delta t\right)-\lambda_{n} a_{n}(M-1) \alpha_{n} \beta_{n} \Delta t^{2}-2 \lambda_{n} \alpha_{n} \beta_{n} \Delta t^{2} \\
-\lambda_{n}(M-1)\left[\beta_{n} \Delta t+\alpha_{n} \Delta t_{n}\left(1-a_{n}\right)\right] \alpha_{n} \Delta t, \\
c_{3 n}=-a_{n} \beta_{n} \Delta t-\lambda_{n}\left(\beta_{n} \Delta t_{n}\right)^{2}-\lambda_{n}(M-1) \beta_{n} \Delta t\left[\beta_{n} \Delta t_{n}+\alpha_{n} \Delta t\left(1-a_{n}\right)\right], \\
c_{4 n}=\left(1-a_{n}\right) \beta_{n} \Delta t-a_{n} \zeta_{n} \Delta t-2 \lambda_{n} \beta_{n} \Delta t \zeta_{n} \Delta t-\lambda_{n}\left[\left(1-a_{n}\right)(M-1) \alpha_{n} \Delta t \beta_{n} \Delta t\right. \\
\left.+(M-1) \zeta_{n} \Delta t\left(\alpha_{n} \Delta t\left(1-a_{n}\right)+\beta_{n} \Delta t\right)\right], \\
c_{5 n}=\left(1-a_{n}\right) \alpha_{n} \Delta t+a_{n} \zeta_{n} \Delta t-2 \lambda_{n} \alpha_{n} \Delta t \zeta_{n} \Delta t-\lambda_{n}\left[\left(1-a_{n}\right)(M-1)\left(\alpha_{n} \Delta t\right)^{2}\right. \\
\left.+a_{n}(M-1) \alpha_{n} \Delta t \zeta_{n} \Delta t\right], \\
c_{6 n}=\left(1-a_{n}\right) \zeta_{n} \Delta t-\lambda_{n}\left(\zeta_{n} \Delta t_{n}\right)^{2}-\lambda_{n}\left(1-a_{n}\right)(M-1) \alpha_{n} \Delta t \zeta_{n} \Delta t_{n} .
\end{array}\right.
$$

The coefficient of the reaction to private information at the $n t h$ auction, $\alpha_{n}$, is equal to:

$$
\alpha_{n} \Delta t=\frac{a_{n}+\lambda_{n}\left(k_{2 n}-k_{5 n}\right)}{\lambda_{n}\left[2+(M-1) a_{n}-2 \lambda_{n}\left(k_{3 n}-\frac{1}{2} k_{4 n}\right)\left(1+a_{n}(M-1)\right)\right]} .
$$

The equation for $\psi_{n}$ is given by:

$$
\psi_{n}=\frac{M a_{n}+M \lambda_{n}\left(k_{2 n}-k_{5 n}\right)}{2+(M-1) a_{n}-2 \lambda_{n}\left(k_{3 n}-\frac{1}{2} k_{4 n}\right)\left(1+a_{n}(M-1)\right)} .
$$

By substituting $a_{n}=\frac{\Sigma_{n}}{\Sigma_{n}+\left(1-\psi_{n}\right) \sigma_{\epsilon}^{2}}$ and $\lambda_{n}^{2}=\frac{\psi_{n} \Sigma_{n}-\frac{\psi_{n}^{2} \sigma_{\epsilon}^{2}}{M}}{\sigma_{u}^{2} \Delta t_{n}}$ and developing the previous equation, we get that $\psi_{n}$ is the solution of the following equation of order six:

$$
\begin{gathered}
\frac{\sigma_{\epsilon}^{2}}{M} \gamma_{5 n} \psi_{n}^{6}+\left(\frac{\sigma_{\epsilon}^{2}}{M} \gamma_{4 n}-\Sigma_{n} \gamma_{5 n}\right) \psi_{n}^{5}+\left(\phi_{5 n}+\frac{\sigma_{\epsilon}^{2}}{M} \gamma_{3 n}-\Sigma_{n} \gamma_{4 n}\right) \psi_{n}^{4}+\left(\phi_{4 n}+\frac{\sigma_{\epsilon}^{2}}{M} \gamma_{2 n}-\Sigma_{n} \gamma_{3 n}\right) \psi_{n}^{3} \\
+\left(\phi_{3 n}+\frac{\sigma_{\epsilon}^{2}}{M} \gamma_{1 n}-\Sigma_{n} \gamma_{2 n}\right) \psi_{n}^{2}+\left(\phi_{2 n}-\Sigma_{n} \gamma_{1 n}\right) \psi_{n}+\phi_{1 n}=0
\end{gathered}
$$

with 


$$
\left\{\begin{array}{l}
\gamma_{1 n}=M^{2}\left(k_{2 n}-k_{5 n}\right)^{2} \frac{\left(\Sigma_{n}+\sigma_{\epsilon}^{2}\right)^{2}}{\sigma_{u}^{2} \Delta t}, \\
\gamma_{2 n}=-2 M\left(k_{2 n}-k_{5 n}\right)\left(\sigma_{\epsilon}^{2}+\Sigma_{n}\right) \frac{M\left(k_{2 n}-k_{5 n}\right) \sigma_{\epsilon}^{2}-2\left(k_{3 n}-\frac{1}{2} k_{4 n}\right)\left(M \Sigma_{n}+\sigma_{\epsilon}^{2}\right)}{\sigma_{u}^{2} \Delta t}, \\
\gamma_{3 n}=\frac{4\left(k_{3 n}-\frac{1}{2} k_{4 n}\right)^{2} \sigma_{\epsilon}^{4}+M^{2}\left(k_{2 n}-k_{5 n}\right)^{2} \sigma_{\epsilon}^{4}+4 M^{2}\left(k_{3 n}-\frac{1}{2} k_{4 n}\right)^{2} \Sigma_{n}^{2}-4 M\left(k_{2 n}-k_{5 n}\right)\left(k_{3 n}-\frac{1}{2} k_{4 n}\right) \Sigma_{n} \sigma_{\epsilon}^{2}}{\sigma_{u}^{2} \Delta t} \\
-\frac{8 M\left(k_{2 n}-k_{5 n}\right) \sigma_{\epsilon}^{4}\left(k_{3 n}-\frac{1}{2} k_{4 n}\right)+4 M^{2}\left(k_{2 n}-k_{5 n}\right) \sigma_{\epsilon}^{2}\left(k_{3 n}-\frac{1}{2} k_{4 n}\right) \Sigma_{n}-8\left(k_{3 n}-\frac{1}{2} k_{4 n}\right)^{2} M \Sigma_{n} \sigma_{\epsilon}^{2}}{\sigma_{u}^{2} \Delta t}, \\
\gamma_{4 n}=\frac{4 M\left(k_{2 n}-k_{5 n}\right)\left(k_{3 n}-\frac{1}{2} k_{4 n}\right) \sigma_{\epsilon}^{4}-8\left(k_{3 n}-\frac{1}{2} k_{4 n}\right)^{2} \sigma_{\epsilon}^{4}-8 M\left(k_{3 n}-\frac{1}{2} k_{4 n}\right)^{2} \Sigma_{n} \sigma_{\epsilon}^{2}}{\sigma_{u}^{2} \Delta t}, \\
\gamma_{5 n}=\frac{4\left(k_{3 n}-\frac{1}{2} k_{4 n}\right)^{2} \sigma_{\epsilon}^{4}}{\sigma_{u}^{2} \Delta t},
\end{array}\right.
$$

and

$$
\left\{\begin{array}{l}
\phi_{1 n}=M^{2} \Sigma_{n}^{2} \\
\phi_{2 n}=-2 M \Sigma_{n}\left(2 \sigma_{\epsilon}^{2}+(M+1) \Sigma_{n}\right) \\
\phi_{3 n}=4 \sigma_{\epsilon}^{4}+4 \sigma_{\epsilon}^{2}(M+1) \Sigma_{n}+(M+1)^{2} \Sigma_{n}^{2}+4 M \sigma_{\epsilon}^{2} \Sigma_{n} \\
\phi_{4 n}=-8 \sigma_{\epsilon}^{4}-4 \sigma_{\epsilon}^{2} \Sigma_{n}(M+1) \\
\phi_{5 n}=4 \sigma_{\epsilon}^{4}
\end{array}\right.
$$

At the final auction there is no future profit, we then have $k_{2 N}=0$ and $k_{3 N}=0$. The parameter $\psi_{N}$ is derived from:

$$
\phi_{5 N} \psi_{N}^{4}+\phi_{4 N} \psi_{N}^{3}+\phi_{3 N} \psi_{N}^{2}+\phi_{2 N} \psi_{N}+\phi_{1 N}=0
$$

The previous equation can be factorized as:

$$
\left[2 \sigma_{\epsilon}^{2} \psi_{N}^{2}-\left(2 \sigma_{\epsilon}^{2}+(M+1) \Sigma_{N}\right) \psi_{N}+M \Sigma_{N}\right]^{2}=0
$$

Hence, the parameter $\psi_{N}$ is derived from:

$$
2 \sigma_{\epsilon}^{2} \psi_{N}^{2}-\left(2 \sigma_{\epsilon}^{2}+(M+1) \Sigma_{N}\right) \psi_{N}+M \Sigma_{N}=0
$$

This ends the proof of proposition 1 .

The case of perfect private information: $\tilde{S}_{i}=\tilde{v}$

We now illustrate our model when $\sigma_{\epsilon}^{2}=0$.

In this case, the demand function of informed trader $i$ at auction $n$ becomes:

$$
\hat{x}_{i n}=\alpha_{n} \Delta t \tilde{v}+\beta_{n} \Delta t \hat{p}_{n-1}^{i},
$$


and his expected profit:

$$
E\left[\pi_{i n} \mid \hat{p}_{0}^{i}, \ldots, \hat{p}_{n-1}^{i}, \tilde{v}\right]=k_{1, n-1} \tilde{v}^{2}+k_{2, n-1} \hat{p}_{n-1}^{i} \tilde{v}+k_{3, n-1} \hat{p}_{n-1}^{i}{ }^{2}+\delta_{n-1} .
$$

$\psi_{n}$ is solution of the following equation:

$$
\begin{gathered}
\frac{4\left(k_{3 n}-\frac{1}{2} k_{4 n}\right)^{2} \Sigma_{n}}{\sigma_{u}^{2} \Delta t_{n}} \psi_{n}^{3}+\left[\frac{4\left(k_{2 n}-k_{5 n}\right)\left(k_{3 n}-\frac{1}{2} k_{4 n}\right) \Sigma_{n}}{\sigma_{u}^{2} \Delta t}-\left(\frac{M+1}{M}\right)^{2}\right] \psi_{n}^{2} \\
+\left(2 \frac{M+1}{M}+\frac{\Sigma_{n}\left(k_{2 n}-k_{5 n}\right)^{2}}{\sigma_{u}^{2} \Delta t}\right) \psi_{n}-1=0 .
\end{gathered}
$$

At the final auction, the parameter $\psi_{N}$ is derived from:

$$
-\left(\frac{M+1}{M}\right)^{2} \psi_{N}^{2}+2 \frac{M+1}{M} \psi_{N}-1=0
$$

then:

$$
\psi_{N}=\frac{M}{M+1}
$$

The coefficients $\alpha_{n}, \beta_{n}, a_{n}$ and $\lambda_{n}$ are characterized by the following equation:

$$
\lambda_{N}=\sqrt{\frac{M}{M+1} \frac{\Sigma_{N}}{\sigma_{u}^{2} \Delta t_{n}} .}
$$

Since $\sigma_{\epsilon}^{2}=0$, we have $a_{n}=1$ for $n=1, \ldots, N$, we then get:

$$
\beta_{N} \Delta t=-\frac{1}{(M+1) \lambda_{N}}=-\alpha_{N} \Delta t
$$

We can prove, by using mathematical induction, that for all auctions $n=1, \ldots, N$ we have:

$$
\begin{gathered}
\beta_{n} \Delta t=-\alpha_{n} \Delta t=-\frac{1-2 \lambda_{n}\left(k_{1 n}-\frac{1}{2} k_{4 n}\right)}{\lambda_{n}\left[M\left(1-2 \lambda_{n}\left(k_{1 n}-\frac{1}{2} k_{4 n}\right)+1\right]\right.}, \\
k_{3 n}=k_{1 n}, \\
k_{2 n}=-2 k_{1 n} .
\end{gathered}
$$

Then, the demand function and the expected profit of informed trader $i$ can be written as:

$$
\begin{gathered}
\hat{x}_{i n}=\alpha_{n} \Delta t\left(\tilde{v}-\hat{p}_{n-1}^{i}\right), \\
E\left[\pi_{i n} \mid \hat{p}_{0}^{i}, \ldots, \hat{p}_{n-1}^{i}, \tilde{v}\right]=k_{1 n-1}\left(\tilde{v}-\hat{p}_{n-1}^{i}\right)^{2}+\delta_{n-1},
\end{gathered}
$$


with

$$
\delta_{n-1}=\delta_{n}+\lambda_{n}^{2} k_{3 n} \sigma_{u}^{2} \Delta t
$$

Furthermore, we have:

$$
\lambda_{n}^{2}=\psi_{n} \frac{\Sigma_{n}}{\sigma_{u}^{2} \Delta t}=M \lambda_{n} \alpha_{n} \Delta t \frac{\Sigma_{n}}{\sigma_{u}^{2} \Delta t} .
$$

Solving the previous expression (6.23) for $\lambda_{n}$ leads to:

$$
\lambda_{n}=\frac{M \alpha_{n} \Sigma_{n}}{\sigma_{u}^{2}}
$$

These equations are similar to those obtained by Holden and Subrahmanyam (1992).

The case of static setting: $N=1$

We now illustrate our model for $N=1$.

In this case, we have $\Delta t=1$ and $\alpha_{N}$ satisfies the following equation:

$$
\alpha_{N}=\frac{a_{N}}{\lambda_{N}\left[2+(M-1) a_{N}\right]} .
$$

We also have:

$$
a_{N}=\frac{\Sigma_{0}}{\Sigma_{0}+\sigma_{\epsilon}^{2}}=\frac{\sigma_{v}^{2}}{\sigma_{v}^{2}+\sigma_{\epsilon}^{2}}=\frac{1}{1+\tau} .
$$

where $\tau=\frac{\sigma_{\epsilon}^{2}}{\sigma_{v}^{2}}$. Then, $\alpha_{N}$ can be written as follows:

$$
\alpha_{N}=\frac{1}{\lambda_{N}(M+1+2 \tau)} \text {. }
$$

This leads to the fact that $\lambda_{N}$ can be written as:

$$
\lambda_{N}=\frac{\sqrt{M}}{M+1+2 \tau} \sqrt{1+\tau} \frac{\sigma_{v}}{\sigma_{u}} .
$$

We then obtain:

$$
\alpha_{N}=\frac{\sigma_{u}}{\sigma_{v}} \frac{1}{\sqrt{M(1+\tau)}}
$$

By substituting $\alpha_{N}$ and $\lambda_{N}$ by their expressions, the value function can be written as follows:

$$
E\left[\pi_{0}^{i}\right]=\frac{\sigma_{u} \sigma_{v} \sqrt{1+\tau}}{\sqrt{M}(M+1+2 \tau)} .
$$

These results are similar to those obtained by Dridi and Germain (2009) when all insiders have the same level of noise in their signals and there is one auction.

\section{All other Propositions are obtained by numerical procedures.}




\section{$7 \quad$ References}

Admati, A., Pfleiderer, P., (1988). Divide and Conquer: A Theory of Intraday and Dayof-the-Week Mean Effects. Review of Financial Studies, 1, 3-40.

Back, K., Cao, H., Willard, A., (2000). Imperfect Competition Among Informed Traders. Journal of Finance, 5, 2117-2155.

Back, K., Pedersen, H., (1998). Long-Lived Information and Intraday Patterns. Journal of Financial Markets 1, 385-402.

Cho, J-W., (2007). Earnings Announcements, Private Information, and Strategic Informed Trading. Journal of Financial Intermediation 16, 117-149.

Dridi, R., Germain, L., (2009). Noise and Competition in Strategic Oligopoly. Journal of Financial Intermediation 18, 311-327.

Ellison, S., Mullin, W., (2007). Gradual Incorporation of Information into Stock Price: An Empirical Study of Informed Trading, Working Paper, MIT.

Foster, F. D., and Viswanathan, S., (1993). The effect of Public Information and Competition on Trading Volume and Price Volatility. Review of Financial Studies, 6, 23-56.

Foster, F. D., Viswanathan, S. (1996). Strategic Trading When Agents Forecast the Forecasts of Others. Journal of Finance 51, 1437-78.

Holden, C. W., Subrahmanyam, A., (1992). Long-Lived Private Information and Imperfect Competition. Journal of Finance 47, 247-270.

Kyle, A., (1985). Continuous Auctions and Insider Trading. Econometrica 53, 1315-1335.

Michener, R., Tighe, C., (1991). A Cournot Model of Insider Trading. Unpublished Working Paper, University of Virginia. 
Ostrovsky, M., (2012).Information Aggregation in Dynamic Markets with Strategic Traders. Econometrica, 80, 2595-2647.

Subrahmanyam, A., (1991). Risk Aversion, Market Liquidity, and Price Efficiency. Review of Financial Studies, 4, 417-441. 